\title{
IL-9-triggered IncRNA Gm13568 regulates Notch1 in astrocytes through interaction with CBP/P300: contribute to the pathogenesis of experimental autoimmune encephalomyelitis
}

Xiaomei Liu ( $\boldsymbol{\nabla}$ lxmlxm_hi@xzhmu.edu )

Xuzhou Medical University https://orcid.org/0000-0003-0607-218X

Feng Zhou

Xuzhou Medical University

Weixiao Wang

Xuzhou Medical University

Guofang Chen

Xuzhou Central Hospital

Qingxiu Zhang

Xuzhou Medical University

Ruixue Lv

Xuzhou Medical University

Zijun Zhao

Xuzhou Medical University

Xiangyang $\mathrm{Li}$

Xuzhou Medical University

Qian Yu

Xuzhou Medical University

Jessica M Meves

University of Michigan

Hui Hua

Xuzhou Medical University

Xiaocui Li

Xuzhou Medical University

Xiaotian Wang

Xuzhou Medical University

Hong Sun

Xuzhou Medical University

Dianshuai Gao

Xuzhou Medical University 


\section{Research}

Keywords: IL-9, astrocytes, IncRNA Gm13568, Notch1, inflammatory cytokines, experimental autoimmune encephalomyelitis (EAE)

Posted Date: February 24th, 2021

DOI: https://doi.org/10.21203/rs.3.rs-249849/v1

License: (c) (i) This work is licensed under a Creative Commons Attribution 4.0 International License. Read Full License 


\section{Abstract \\ Background}

Interleukin 9 (IL-9), produced mainly by T helper 9 (Th9) cells, has been recognized as an important regulator in multiple sclerosis (MS) and its animal model, experimental autoimmune encephalomyelitis (EAE). Astrocytes respond to IL-9 and reactive astrocytes always associate with blood-brain barrier damage, immune cells infiltration and spinal injury in MS and EAE. Several long non-coding RNAs (IncRNAs) with aberrant expression have been identified in the pathogenesis of MS. Here, we examined the effects of IncRNA Gm13568 (a co-upregulated IncRNA both in EAE mice and in mouse primary astrocytes activated by IL-9) on the activation of astrocytes and the process of EAE.

\section{Methods}

In vitro, shRNA-recombinant lentivirus with Glial fibrillary acidic protein (GFAP) promoter were performed to determine the relative gene expression and proinflammatory cytokines production in IL-9 treatedastrocytes using Western blot, real-time PCR and Cytometric bead array, respectively. RIP and ChIP assays were analyzed for the mechanism of IncRNA Gm13568 regulating gene expression. Immunofluorescence assays was performed to measure the protein expression in astrocytes. In vivo, H\&E staining and LFB staining were applied to detect the inflammatory cells infiltrations and the medullary sheath damage in spinal cords of EAE mice infected by the recombinant lentivirus. Results were analyzed by one-way ANOVA or student's t-test, as appropriate.

\section{Results}

Knockdown of the endogenous IncRNA Gm13568 remarkably inhibits the Notch1 expression, astrocytosis and the phosphorylation of signal transducer and activator of transcription 3 (p-STAT3) as well as the production of inflammatory cytokines and chemokines (IL-6, TNF-a, IP-10) in IL-9 activated astrocytes. In which, Gm13568 associates with CBP/P300 is enriched in the promoter of Notch1 genes. More importantly, inhibiting Gm13568 with lentiviral vector in astrocytes ameliorates significantly inflammation and demyelination in EAE mice, therefore delaying the EAE process.

\section{Conclusions}

These findings uncover that $\mathrm{Gm} 13568$ regulates the production of inflammatory cytokines in active astrocytes and affects the pathogenesis of EAE through the Notch1/STAT3 pathway. LncRNA Gm13568 may be a promising target for treating MS and demyelinating diseases.

\section{Background}


Multiple sclerosis (MS) is a chronic inflammatory autoimmune disease of the central nervous system (CNS), in which immune cells infiltrate into the white matter with demyelination and axonal loss [1]. The occupancy of T cells and glia cells in patient lesions suggests that both are important contributors to MS $[2,3]$. Autoreactive effector $C D 4^{+} T$ cells, including proinflammatory Th1 cell and Th17 cell, are believed as the major effector cells [4], which migrate into the CNS and initiate MS process. Recently, Th9 cell and its secreting cytokine IL-9 have been implicated in MS, which activates and cooperates with other $\mathrm{CD} 4^{+} \mathrm{T}$ cells and CNS-resident cells in MS and experimental autoimmune encephalomyelitis (EAE), an animal model of MS [5].

Astrocytes, the most abundant cell population in the CNS, play an essential role in the homeostasis of CNS. Astrocytes participate in the formation and integrity of the blood-brain barrier (BBB), support of neurons and regulation of axonal outgrowths. When CNS injuries occur, astrocytes become activated, which are clearly identified by the dramatically upregulated expression of glial fibrillary acidic protein (GFAP) [6, 7]. In response to IL-9 stimulation, astrocytes aggravate the BBB disruption, increase chemokines production and facilitate T cell migration into the CNS $[8,9]$. However, the mechanisms by which astrocyte activation with IL-9 stimulation contribute to MS remains undefined.

The Notch signaling is a fundamental and well-conserved pathway associated with cell-fate. The canonical Notch pathway arises from the interaction of transmembrane Notch receptors (Notch1-4) with ligands (Jagged1, Jagged2, DLL1, DLL3 or DLL4), and non-canonical pathway is activated through various molecules, such as NF-KB, STAT3 $[10,11]$. Upon ligand binding or activation, Notch1 is cleaved and releases Notch1 intracellular domain (NICD) that directly translocates into the nucleus to form a complex with RBP/J and mastermind like (MAML) proteins, which promotes the transcription of target genes $[10,11]$. In addition, NICD binds to the serine residue of phosphorylated STAT3 (p-STAT3), facilitates the nuclear translocation of p-STAT3 and initiates the transcription of corresponding target genes $[12,13]$. It has been reported that Notch is associated with several progressive neurodegenerative diseases, including MS, amyotrophic lateral sclerosis (ALS) and Alzheimer's disease (AD). Notch1 signaling activation increases astrocyte proliferation and enhances reactive astrogliosis after CNS injury $[14,15]$.

Long noncoding RNAs (IncRNAs), ranging from hundreds to tens of thousands of nucleotides in length with no protein-coding capacity, have been proved to play vital roles in diverse biological processes of genomic imprinting and development to immune response [16]. LncRNAs are involved in neurodegenerative diseases, including MS, ALS, AD, Parkinson's disease (PD) and Huntington's disease (HD) $[17,18]$. Importantly, differentially expressed-IncRNAs in the activated astrocytes are closely relative to EAE progression [19]. However, the mechanisms of IncRNAs regulating astrocytes activation and MS process are still poorly understood.

The transcriptional co-activators CREB-binding protein (CBP) and p300 are histone acetyltransferase (HAT) contributing to histone 3 lysine 27 acetylation (H3K27ac), RNA Pol II acetylation and NF-KB enrichment in the promoter region of target genes, which forms a transcription network to facilitate the 
expression of target gene [20-24]. Lately, it has been reported that CBP/P300 binds directly to RNA to enhance histone acetylation and gene transcription [25]. Additionally, IncRNAs are associated with the CBP/p300 complex and RNA polymerase II, which regulate gene expression $[24,26]$.

In previous study, we analyzed IncRNA expression profiles in EAE mice as well as in activated astrocytes with IL-9 stimulation [19]. Here, we aim to further explore the function and mechanism of IncRNA Gm13568 (a co-upregulated IncRNA in EAE mice and IL-9-activated astrocytes) in regulating astrocytes activation and EAE progression. We display that $\mathrm{Gm} 13568$ contributes to the production of inflammatory cytokines and chemokines in activated astrocytes through interaction with CBP/P300, which is enriched in the promoter of Notch 1 and promotes Notch 1 expression and signaling activation, thereby aggravating the EAE damage. These results suggest that targeting IncRNAs in astrocytes is an attractive option for the treatment of MS.

\section{Methods}

\section{Animal, antibodies and reagents}

C57BL/6 mice were purchased from the Shanghai Experimental Animal Center, Chinese Academy of Sciences. All mice were bred under specific pathogen-free conditions. Antibodies used in this study were: anti-IL-9 antibody (Rabbit, ab203386, Abcam), anti-GFAP antibody (Rabbit, ab7260, Abcam), anti-GFAP (Mouse, ab4648, Abcam), anti-cleaved Notch1 antibody (NICD, Rabbit, 4147, Cell Signaling Technology), anti-Notch 1 antibody (Goat, sc-6014, SANTA CRUZ), anti-p-STAT3 (Rabbit, Tyr705, BS4181, Bioworld Technology), anti-STAT3 (Rabbit, AP0365, Bioworld Technology), NF-kB p65 (Rabbit, 8242, Cell Signaling Technology), anti- CBP/P300 antibody (Rabbit, 4771s, Cell Signaling Technology), anti-RNA Pol II antibody (Mouse, 17-620, Sigma Aldrich), anti-H3K27ac antibody (Rabbit, ab177178, Abcam). The secondary antibodies were all purchased from Sigma Biotechnology. Myelin oligodendrocyte glycoprotein (MOG) amino acids 35-55 ( MOG $_{35}-55$ peptides, MEVGWYRSPFSRVVHLYRNGK) was purchased from China Peptides Co. Ltd (shanghai, China). Recombinant mouse IL-9 was from R\&D Systems, Multiplex magnetic bead-based antibody detection kits (cytokine and chemokine detection kits, Cytometric Bead Array) were from BD Biosciences.

\section{EAE induction and clinical evaluation}

C57BL/ 6 female mice (6-8 weeks-old) were immunized with $250 \mu \mathrm{g} \mathrm{MOG}_{35}$ - 55 emulsified in complete Freund's adjuvant (Sigma Aldrich) containing $5 \mathrm{mg} / \mathrm{ml}$ heat-killed Mycobacterium tuberculosis (H37Ra strain, Difco). On day 0 and day 2, mice were intraperitoneally received pertussis toxin ( $200 \mathrm{ng}$, Invitrogen), then mice were monitored daily for clinical signs of EAE according to the following grading scale in a double-blinded manner: 0 , no clinical signs; 1 , limp tail; 2 , paraparesis (weakness, incomplete paralysis of one or two hind limbs); 3, paraplegia (two hind limbs paralysis); 4, paraplegia with fore limb weakness or paralysis; 5 , moribund or death. The PBS with the same volume was injected into mice as the negative control. 


\section{Primary astrocyte culture}

Primary mouse astrocytes were separated and cultured as described previously [27]. Astrocytes were synchronized with non-serum culture media for $12 \mathrm{~h}$ before IL-9 stimulation.

\section{Real-time PCR assay}

Total RNAs from the spinal cords of mouse or astrocytes were extracted by Trizol reagent (Invitrogen). Real-time PCR assay was conducted as described previously [28]. The relative genes transcription was calculated with the $2^{-\triangle \triangle C T}$ method. The primers are listed in Supplementary Table S1.

\section{Cytometric Bead Array (CBA) assay}

The serum of mice or the supernatant of cultured astrocytes was collected. The secretion levels of IL-6, TNF-a, interferon-inducible protein-10 (IP-10) and monocyte chemoattractant protein-1 (MCP-1) were detected by CBA assay (BD Biosciences, USA) as described previously [29].

\section{Western blot assay}

As described previously [27], the total protein was extracted from the spinal cords of mouse as well as the cultured astrocytes. The expression level of protein in samples was normalized by $\beta$-actin protein.

\section{Lentivirus generation}

Recombinant lentiviral vectors carrying with EGFP and with astrocyte-specific promoter of glial fibrillary acidic protein (GFAP) were constructed by Gene Chem (Shanghai, China) and were verified (Supplementary Fig. S1). Because of the sequence of Gm13568 completely complements the sense chain at 9075-9497 locus of Notch1 gene, conventional shRNA knockdown may simultaneously affect the expression of endogenous Notch1 gene. We constructed recombinant lentivirus vectors with GFAP promoter that overexpresses the 9075-9497 sequence of Notch1 gene to make them competitively bind to Gm13568 (LV-Inhibit-Gm13568), so as to inhibit the effect of endogenous Gm13568 on Notch1 expression.

\section{RNA Immunoprecipitation (RIP) assay}

RIP assay was performed as previously described [30]. In brief, after treating with IL-9, $2 \times 10^{7}$ primary mouse astrocytes were treated with $0.3 \%$ formaldehyde in culture medium, then $0.125 \mathrm{M}$ Glycine was added to the medium. Cells were washed in cold PBS and pelleted. The pellets were resuspended in RIPA buffer and incubated on ice, and the lysate was centrifugated at 13,000 RPM for 10 min. Antibodies mixing Protein $\mathrm{A} / \mathrm{G}$ beads were added and incubated at $4^{\circ} \mathrm{C}$ overnight. Next, the beads were resuspended in RIPA buffer and were treated with proteinase $\mathrm{K}$ at $45^{\circ} \mathrm{C}$ for $45 \mathrm{~min}$. Finally, samples were extracted with Trizol and then RNA was detected by real-time PCR assay. The data of retrieved RNAs were calculated by the subtraction of RT/input ratio and non-RT/input ratio. The primer sequence of Gm13568 was listed in Supplementary Table S1.

\section{Chromatin Immunoprecipitation (ChIP) assay}


ChIP assays were performed as described previously [30]. Primary mouse astrocytes were treated with IL9 for 6 h. $1 \times 10^{7}$ cells were used for each ChIP-enrichment. Chromatin was sheared to the fragment size of 200-500 bp. The antibodies were anti-NF-kB p65, anti-H3K27ac, anti-RNA Pol II, anti-CBP/P300, normal mouse IgG. All immunoprecipitated chromatin DNA was analyzed by real-time PCR. The primer sequences were listed in Supplementary Table S1.

\section{Histopathology assay}

Histopathology assay was performed as previously described [27, 31]. Hematoxylin and eosin (H\&E) or luxol fast blue (LFB) staining on $4 \mu \mathrm{m}$ paraffin-embedded spinal cord sections were used in evaluating inflammation and demyelination, respectively.

\section{Statistical analysis}

Statistical analysis was performed by GraphPad Prism version 5.0 software with the two-tailed unpaired Student's $t$ test or one-way multiple-range analysis of variance (ANOVA). A Mann-Whitney test was used for nonparametric data (EAE scoring). Data presented as mean \pm standard error of mean (SEM), calculated for all points from at least three independent experiments in triplicates. Values of $P<0.05$ was considered significant.

\section{Results}

The production of IL-9 and inflammatory cytokines as well as the expression of NICD and p-STAT3 are increased in EAE mice

It has been recently demonstrated that Th9/IL-9 is a key player in regulating autoimmune responses in MS and EAE accompanied by the production of a large number of inflammatory cytokines. To investigate the potential role of IL-9 in inducing Notch1 pathway activation, reactive astrogliosis and the production of inflammatory cytokines in EAE mice, we firstly measured the expression of IL-9 in the tissues of spinal cords. As shown in Fig. 1a, the mRNA and protein level of IL-9 was gradually increased in the spinal cords along with the severity of EAE. Then, the expression of GFAP, NICD and p-STAT3 was detected by Western blot assay. The results showed that the expression of GFAP protein was markedly elevated in the spinal cords of EAE mice with score 2.0 and lasted until score 4.0. Similarly, the expression of NICD and the phosphorylation level of STAT3 (p-STAT3) was also significantly upregulated from EAE score 2.0 to score 4.0 (Fig. 1b). Finally, the mRNA transcription and secretion of pro-inflammatory cytokines and chemokines (IL-6, TNF-a, IP-10, and MCP-1) in the spinal cords and peripheral blood of EAE mice were assessed by real-time PCR and CBA assay, respectively (Fig. 1c, d). The production of IL-6, TNF-a, IP-10 and MCP-1 was obviously increased along with EAE process, which was consistent with our previous study [27]. These results suggest whether IL-9 secreted by Th cells stimulates astrogliosis and Notch1 signal activation, therefore promoting the production of pro-inflammatory cytokines and chemokines in the CNS of EAE mice. 


\section{IL9 stimulates astrocytes to promote Notch1 pathway activation and inflammatory cytokine production}

To explore the possible roles of IL-9 in Notch1 pathway activation and inflammatory cytokines production in astrocytes, primary mouse astrocytes were treated with IL-9 in serum-free media. Western blot results showed that the expression of GFAP was dramatically increased at $6 \mathrm{~h}$ and lasted for $24 \mathrm{~h}$ in the astrocytes with IL-9 treatment. Meanwhile, the expressions of NICD and p-STAT3 were elevated at $6 \mathrm{~h}$ after IL-9 stimulation (Fig. 2a). The results of immunofluorescence assay indicated that the expression of Notch1 in cytoplasm was also significantly increased in IL-9 stimulated-astrocytes, compared to the DMEM group (Fig. 2b). In addition, the mRNA transcription and secretion levels of inflammatory cytokines such as IL-6, TNF-a, IP-10, and MCP-1 were simultaneously upregulated and peaked at $6 \mathrm{~h}$ in the astrocytes stimulated by IL-9 (Fig. 2c, d). These data suggest that IL-9 activates Notch1/ STAT3 signaling pathway and promotes the productions of pro-inflammatory cytokines in astrocytes.

\section{Knockdown Notch1 suppresses inflammatory response in astrocytes by IL-9}

To further verify whether Notch1 is involved in regulating inflammatory responses in IL-9 stimulatedastrocytes, recombinant lentiviral vector of three Notch1-shRNA (LV-Notch1-shRNA) specifically targeting astrocytes was constructed, and then transfected into primary mouse astrocytes, respectively. As shown in Fig. 3a, Notch1 protein level was significantly repressed in Notch1-shRNA-1, shRNA-2 and shRNA-3 group, particularly in shRNA-3 group, compared with shRNA-ctrl group. So, the recombinant lentiviral vector of Notch1-shRNA-3 was selected for packaging and infection. Western blot results showed that the expression of NICD and the phosphorylation level of STAT3 were obviously decreased from LV-Notch1shRNA infection group in IL-9 treated-astrocytes, compared to the LV-ctrl group (Fig. 3b). Furthermore, the production of inflammatory cytokines including IL-6, TNF-a and IP-10 was markedly reduced in LVNotch1-shRNA infected-astrocytes (Fig. 3c, d). These results indicate that IL-9-induced upregulation and activation of Notch1 contribute to the inflammatory cytokines production in astrocytes.

\section{Identification of IncRNA Gm13568 targeting Notch1}

In our previous study, the changes of IncRNA profiles were reported both in EAE mice (in vivo) and in activated astrocytes with IL-9 stimulation (in vitro) [19]. We further found that the sequence of ENSMUST00000156099 (Gm13568, one of IncRNA co-upregulated in vivo and in vitro,) with 446bp length, is identical to the antisense sequence of Notch1 gene at 9075-9497 locus that is complementary to the sense chain of Notch 1 gene (located between exon 2 and exon 3) (Fig. 4a, b). The time course data showed that the changes of mRNA level between Gm13568 and Notch1 reflected the same tendency. At 3 h of IL-9 treatment, the levels of Gm13568 and Notch1 were significantly increased, and lasted for $12 \mathrm{~h}$ (Fig. 4c). Taken together, these results suggest that Gm13568 may have a positive regulatory effect on Notch1 expression. 


\section{LncRNA Gm13568 regulates Notch1 pathway and inflammatory cytokines production in activated astrocytes by IL-9}

To evaluate the roles of Gm13568 in regulating Notch1 expression and signal activation and inflammatory cytokines production in activated astrocytes, we constructed recombinant lentivirus of inhibiting Gm13568 (LV-Inhibit Gm13568), packaged and infected primary mouse astrocytes. In LVInhibit-Gm13568 group, the expressions of GFAP and Notch1were in lower level after IL-9 stimulation for $6 \mathrm{~h}$ than that of LV-ctrl group, indicating the inhibition of Gm13568 significantly decreased the Notch1 expression and reactive astrocytosis (Fig. 5a). Similarly, the expression of NICD and p-STAT3 were also markedly decreased in LV-Inhibit-Gm13568 group (Fig. 5a). Additionally, the production of IL-6, TNF-a and IP-10 were in much lower level in LV-Inhibit-Gm13568 group (Fig. 5b, c). These observations indicate that Gm13568 regulates the expression and signal activation of Notch1, therefore mediating the production of inflammatory cytokines in astrocytes.

\section{The interaction of Gm13568 with CBP/P300 regulates the Notch1 expression in astrocytes by IL-9}

To further explore the potential regulating mechanism of Gm13568 on Notch1 expression, RIP assay was performed to analyze Gm13568 and NF-kB p65 or CBP/P300 interaction. As shown in Fig. 6a, Gm13568 was significantly interacted with NF-KB p65 and CBP/P300 in activated astrocytes by IL-9. Moreover, ChIP assay revealed that NF-KB p65, CBP/P300, RNA Pol II, and H3K27ac were differentially enriched in the Notch1 gene promoter in IL-9 activated astrocytes (Fig. 6b). Importantly, inhibition of Gm13568 significantly reduced NF-KB p65 and CBP/P300 enrichment in the Notch1 promoter (Fig. 6c). The data indicate that the interaction of Gm13568 with CBP/P300 may epigenetically regulate the transcription of Notch1 gene.

\section{Notch1 knockdown in astrocytes represses inflammation and alleviates EAE in mice}

To further clarify the role of Notch1 from astrocytes in EAE development, $1 \times 10^{7}$ transforming units of recombinant lentivirus of LV-Notch1-shRNA specifically targeting astrocytes was injected into mice via the tail vein. After 7 days, EAE was induced by $M O_{35-55}$ for 23 days. The clinical score showed that silencing Notch1 in astrocytes not only delayed the onset of EAE but also relieved illness (Fig. 7a). Meanwhile, the production of IL-9 was obviously decreased and the expression of GFAP was significantly depressed in EAE mice with LV-Notch1-shRNA (Fig. 7b). Furthermore, the NICD and p-STAT3 protein expressions were also markedly downregulated in the spinal cords of LV-Notch1-shRNA group (Fig. 7b). The levels of inflammatory cytokines such as IL-6, TNF-a and IP-10 were reduced in LV-Notch1-shRNA group (Fig. 7c, d). More importantly, H\&E and LFB staining strongly displayed that LV-ctrl EAE mice had 
large number of inflammatory cell infiltration and more severe demyelination lesion in the spinal cords. However, the EAE mice with LV-Notch1-shRNA had less inflammatory cell infiltration and slight demyelination lesion in the spinal cords (Fig. 7e, f). Collectively, these data reveal that Notch1 participants in the activation and hyperplasia of astrocytes, resulting in aggravation of inflammatory cell infiltration and spinal cords injury in EAE mice, which may be associated with an increased inflammatory reaction. On the contrary, knockdown Notch1 reversed these results above.

\section{The inhibition of Gm13568 in astrocytes decreases the activation of Notch1 pathway and ameliorates EAE process in mice}

To verify whether Gm13568 regulating Notch1 in astrocytes contributes to the development of EAE, $1 \times 10^{7}$ transforming units of recombinant lentivirus targeting astrocytes (LV-Inhibit-Gm13568 or LV-ctrl) was administrated to mice via the tail vein, and then EAE was induced after 7 days. As shown in Fig. 8a, inhibiting Gm13568 in astrocytes slowed down the EAE development. At the same time, the production of IL-9 was significantly reduced compared to the LV-ctrl mice (Fig. 8b). The expressions of GFAP, Notch1, NICD and p-STAT3 were dramatically decreased in LV-Inhibit-Gm13568 mice (Fig. 8b). Similarly, the production of IL-6, TNF- $a$ and IP-10 was also markedly diminished in LV-Inhibit-Gm13568 group (Fig. 8c, d). In addition, H\&E and LFB staining showed that inflammatory cell infiltration and demyelination lesion in white matter were obviously ameliorated in LV-Inhibit-Gm13568 group, compared to the LV-ctrl mice (Fig. 8e, f). Taken together, these data imply that IncRNA Gm13568 regulates Notch1 pathway in astrocytes, thus controlling the reactive astrocytosis, inflammatory cytokines secretion and spinal cord injury in mice, and in turn affecting the EAE process.

\section{Discussion}

Astrocytes play important roles in MS by recruiting lymphocytes, confining inflammation and contributing to tissue damage [31, 32]. It has also been reported that IncRNAs have significant impacts on normal neural development and the progression of neurodegenerative diseases [17]. Our previous studies have demonstrated that IncRNAs differentially expressed in the activated astrocytes are closely related to EAE process [19]. Here, we confirm that inhibiting IncRNA Gm13568, an upregulated IncRNA both in IL9activated astrocytes and in EAE mice, reduces inflammatory cytokines production in astrocytes and delays EAE process through decreasing Notch1 expression and signal activation by interaction with NFKB p65 and CBP/P300 (Fig. 9).

In the CNS autoimmunity, reactive astrocytes can form the detrimental positive-feedback loop of inflammation, which accelerates the inflammatory cascades and aggravates neurological disorders [3]. Response to inflammatory stress, many genes relevant to inflammation, oxidative stress, immune receptors and BBB disruption, are increased in astrocytes [33]. It is demonstrated that astrocytes aggravate the BBB disruption, increase chemokines production and facilitate $T$ cell migration to the CNS upon IL-9 stimulation [8, 9]. Our data showed that the mRNA transcription and protein expression of IL-9 in the spinal cords of EAE mice were gradually increased along with the severity of EAE. Notably, the 
expression of GFAP and the secretion of pro-inflammatory cytokines were also significantly upregulated along with the severity of EAE, which implied the activation and hyperplasia of reactive astrocytes. However, the understanding of IL-9 promoting astrogliosis is still very limited.

Notch pathway can modulate immune response and inflammatory processes, which are clinically critical in MS. Emerging evidence indicates the Notch signal regulates the development and maturation of microglia, oligodendrocytes and $C D 4^{+} T$ cells in MS lesions, which are associated with MS pathology [34]. Inhibiting Notch1 pathway in oligodendrocytes improved remyelination in EAE mice and cuprizone (CPZ) demyelination model $[34,35]$. It is demonstrated that Notch-1 signaling is a critical regulator of intracerebral hemorrhage (ICH)-induced reactive astrogliosis [15]. Our results showed that the expression of Notch1, NICD and p-STAT3 was consistent with that of GFAP and inflammation cytokines production in EAE and in reactive astrocytes with IL-9 stimulation. Importantly, knockdown Notch1 in astrocytes not only decreased inflammatory production both in vivo and in vitro, but also reduced inflammatory cell infiltration and demyelination lesion in the spinal cords of EAE mice. Yet, the underlying mechanisms of Notch1 modulation on astrocytes are far from clear in MS.

In recent years, IncRNAs having been shown to play vital roles in diverse biological processes from development to immune responses. In CNS, they diversely participate in the neuroinflammation, synapse plasticity, synaptogenesis and memory formation [30,36,37]. Accumulating evidence has indicated that IncRNAs as key regulatory molecules are involved in MS pathogenesis [38-42], but these data mainly focused on the regulation of Th cells and microglia by IncRNAs. Given that astrocytes play essential roles in MS process, it is crucial to understand the mechanism exerted by astrocytes. To date, the functional characterization of IncRNAs during astrocyte activation and MS process has not been fully uncovered. Our previous study identified the changes of IncRNA expression profiles both in EAE mice and in activated astrocytes with IL-9 stimulation [19]. We found that the sequence of co-upregulated IncRNA Gm13568 in vivo and in vitro were complementary to 9075-9497 locus of the sense chain in Notch1 gene, and the time course of Gm13568 changes was consistent with that of Notch 1 in IL-9-stimulated astrocytes. As it is known, IncRNAs occupy the abilities to control the expression of genes as well as contribute to the pathogeneses of diseases. So, we suppose that $\mathrm{Gm} 13568 \mathrm{~m} /$ ay positively regulate the expression of Notch1 gene in astrocytes, therefore aggravating the EAE pathogeneses. In this study, we prove this hypothesis. Our study revealed that the inhibition of Gm13568 decreased Notch 1 expression and pathway activation in astrocytes, as well as attenuated reactive astrocytosis both in vitro and in vivo, which elicited the reduction of inflammatory cytokines and chemokines production, thus alleviated inflammatory cell infiltration and demyelination lesion in EAE mice. However, it is still not very clear about the mechanisms by which IncRNAs act on Notch1 in astrocytes.

It has been demonstrated that IncRNAs can modulate protein-coding gene expression in chromatin remodeling and histone modification, which are always associated with the CBP/p300 complex, the CoREST/REST complex and the Polycomb Repressive Complex 2 (PRC2) complex [16, 36]. In the present study, RIP and ChIP assay showed that Gm13568 associated with NF-kB p65 and CBP/P300 was 
enriched in the promoter of Notch1gene. The data suggest that the interaction of Gm13568 and CBP/P300 might epigenetically regulate Notch1 expression in activated astrocytes and EAE mice.

\section{Conclusion}

In summary, our present evidence reveals that IncRNA Gm13568 induced by IL-9 in astrocytes regulates Notch1 expression through the interaction with CBP/P300 and then promotes Notch1 pathway activation, which is involved in the production of inflammatory cytokines and chemokines in astrocytes, in turn eventually affecting the EAE development. Thus, these findings emphasize that the IncRNA derives from astrocytes may play key roles in neuroinflammation and pathogenesis of MS.

\section{Abbreviations}

IL-9: interleukin-9; CBP/P300: CREB-binding protein (CBP) and p300; MS: multiple sclerosis; EAE: experimental autoimmune encephalomyelitis; STAT3: signal transducer and activator of transcription 3; IL-6: interleukin-6; TNF-a: Tumor necrosis factor-a; IP-10: interferon-gamma induced protein 10; MCP-1: monocyte chemoattractant protein-1; CNS: the central nervous system; GFAP: glial fibrillary acidic protein; NICD: Notch1 intracellular domain; MOG: Myelin oligodendrocyte glycoprotein; CBA: Cytometric Bead Array; RIP: RNA Immunoprecipitation; ChIP: Chromatin Immunoprecipitation.

\section{Declarations}

\section{Acknowledgements}

We would like to thank the professors Kuiyang Zheng and Renxian Tang from the Jiangsu Key Laboratory of Immunity and Metabolism.

\section{Author Contributions}

X.Liu, F. Zhou, W. Wang and D. Gao designed research; W. Wang, X. Li, Z. Zhao and H. Hua performed research; X. Liu, G. Chen, Q. Zhang Y. Yu, X. Li and X. Wang analyzed data; X. Liu, F. Zhou, R. Lv, Jessica M. Meves, H. Sun and D. Gao drafted and revised the paper.

\section{Funding}

This work was supported by the National Natural Science Foundation of China (81971179 to Liu, 82071304 and 81671149 to Zhang, 81702027 to Yu, 81461138036 to Sun), the Natural Science Foundation of Jiangsu Province (BK20191463 to Liu), Jiangsu Commission of Health (Z2019035 to Zhou), Jiangsu Provincial Department of Education (20KJA320004 to Zhou), the Priority Academic Program Development of Jiangsu Higher Education Institutions (2017 PAPD), Xuzhou Medical Young Talents Project and Xuzhou Technology Bureau Foundation (KC17115, KC16SS081, KC19167 to Zhang). 


\section{Ethics approval}

Experimental procedures were approved by the Institutional Animal Use Committee of the Shanghai Institutes for Biological Sciences (Chinese Academy of Sciences) and the Ethical Committee for Xuzhou Medical University.

\section{Consent for publication}

Not applicable.

\section{Declaration of Competing Interest}

None of the authors have any conflicts of interest in this study.

\section{Availability of data and material}

All data generated or analyzed during this study are included in this published article and its supplementary information files.

\section{Author details}

${ }^{1}$ Jiangsu Key Laboratory of Immunity and Metabolism, Department of Pathogen Biology and Immunology and Laboratory of Infection and Immunity, Xuzhou Medical University, Xuzhou, Jiangsu 221004, China. ${ }^{2}$ Neurology Department, The Affiliated Xuzhou Center Hospital of Nanjing University of Chinese Medicine, Xuzhou, China; Neurology Department, Xuzhou Central Hospital, Xuzhou, China; Neurology Department, Xuzhou Clinical School of Xuzhou Medical University, Xuzhou, Jiangsu 221009, China. ${ }^{3}$ Department of Neurology, Second Affiliated Hospital of Xuzhou Medical University, Xuzhou Jiangsu 221006, China. ${ }^{4}$ Department of Psychiatry, University of Michigan Medicine, Michigan, MI48109, USA. ${ }^{5}$ Department of Physiology, Xuzhou Medical University, Xuzhou, Jiangsu 221004, P.R. China. ${ }^{6}$ Department of Neurobiology and Anatomy, Xuzhou Medical University, Xuzhou, Jiangsu 221004, China.

\section{References}

1. Faissner S, Plemel JR, Gold R, Yong VW. Progressive multiple sclerosis: from pathophysiology to therapeutic strategies. Nat Rev Drug Discov. 2019;1812:905-22.

2. Dong Y, Yong VW. When encephalitogenic T cells collaborate with microglia in multiple sclerosis. Nat Rev Neurol. 2019;1512:704-17.

3. Yi W, Schlüter D, Wang X. Astrocytes in multiple sclerosis and experimental autoimmune encephalomyelitis: Star-shaped cells illuminating the darkness of CNS autoimmunity. Brain Behav Immun. 2019;80:10-24.

4. Baecher-Allan C, Kaskow BJ, Weiner HL. Multiple Sclerosis: Mechanisms and Immunotherapy. Neuron. 2018; 974:742-68. 
5. Elyaman W, Khoury SJ. Th9 cells in the pathogenesis of EAE and multiple sclerosis. Semin Immunopathol. 2017;391:79-87.

6. Brambilla R. The contribution of astrocytes to the neuroinflammatory response in multiple sclerosis and experimental autoimmune encephalomyelitis. Acta Neuropathol. 2019;1375:757-83.

7. Santello $\mathrm{M}$, Toni $\mathrm{N}$, Volterra A. Astrocyte function from information processing to cognition and cognitive impairment. Nat Neurosci. 2019;222:154-66.

8. Tan S, Shan Y, Lin Y, Liao S, Zhang B, Zeng Qdet al. Neutralization of interleukin-9 ameliorates experimental stroke by repairing the blood-brain barrier via down-regulation of astrocyte-derived vascular endothelial growth factor-A. Faseb j. 2019;333:4376-87.

9. Zhou Y, Sonobe Y, Akahori T, Jin S, Kawanokuchi J, Noda M『et al. IL-9 promotes Th17 cell migration into the central nervous system via CC chemokine ligand-20 produced by astrocytes. J Immunol. 2011;1867:4415-21.

10. Arumugam TV, Baik SH, Balaganapathy P, Sobey CG, Mattson MP, Jo DG. Notch signaling and neuronal death in stroke. Prog Neurobiol. 2018;165-167:103-16.

11. Pagie S, Gérard N, Charreau B. Notch signaling triggered via the ligand DLL4 impedes M2 macrophage differentiation and promotes their apoptosis. Cell Commun Signal. 2018;161:4.

12. Nonneman A, Criem N, Lewandowski SA, Nuyts R, Thal DR, Pfrieger FW囚et al. Astrocyte-derived Jagged-1 mitigates deleterious Notch signaling in amyotrophic lateral sclerosis. Neurobiol Dis. 2018;119:26-40.

13. Bhoopathi P, Chetty C, Dontula R, Gujrati M, Dinh DH, Rao JS『et al. SPARC stimulates neuronal differentiation of medulloblastoma cells via the Notch1/STAT3 pathway. Cancer Res. 2011;7114:4908-19.

14. Ho DM, Artavanis-Tsakonas S, Louvi A. The Notch pathway in CNS homeostasis and neurodegeneration. Wiley Interdiscip Rev Dev Biol. 2020;91:e358.

15. Zhong JH, Zhou HJ, Tang T, Cui HJ, Yang AL, Zhang QM『et al. Activation of the Notch-1 signaling pathway may be involved in intracerebral hemorrhage-induced reactive astrogliosis in rats. $J$ Neurosurg. 2018;1293:732-39.

16. Kopp F, Mendell JT. Functional Classification and Experimental Dissection of Long Noncoding RNAs. Cell. 2018;1723:393-407.

17. Wan P, Su W, Zhuo Y. The Role of Long Noncoding RNAs in Neurodegenerative Diseases. Mol Neurobiol. 2017;543:2012-21.

18. Zhang F, Gao C, Ma XF, Peng XL, Zhang RX, Kong DX『et al. Expression Profile of Long Noncoding RNAs in Peripheral Blood Mononuclear Cells from Multiple Sclerosis Patients. CNS Neurosci Ther. 2016;224:298-305.

19. Liu X, Zhang Q, Wang W, Zuo D, Wang J, Zhou F『et al. Analysis of Long Noncoding RNA and mRNA Expression Profiles in IL-9-Activated Astrocytes and EAE Mice. Cell Physiol Biochem. 2018;455:198698. 
20. Dutto I, Scalera C, Prosperi E. CREBBP and p300 lysine acetyl transferases in the DNA damage response. Cell Mol Life Sci. 2018;758:1325-38.

21. Schröder S, Herker E, Itzen F, He D, Thomas S, Gilchrist DA囚et al. Acetylation of RNA polymerase II regulates growth-factor-induced gene transcription in mammalian cells. Mol Cell. 2013;523:314-24.

22. Jin Q, Yu LR, Wang L, Zhang Z, Kasper LH, Lee JE囚et al. Distinct roles of GCN5/PCAF-mediated $\mathrm{H} 3 \mathrm{~K} 9 \mathrm{ac}$ and CBP/p300-mediated H3K18/27ac in nuclear receptor transactivation. Embo j. 2011;302:249-62.

23. Dyson HJ, Wright PE. Role of Intrinsic Protein Disorder in the Function and Interactions of the Transcriptional Coactivators CREB-binding Protein (CBP) and p300. J Biol Chem. 2016;29113:671422.

24. Verhoeven RJA, Tong S, Mok BW, Liu J, He S, Zong J『et al. Epstein-Barr Virus BART Long Non-coding RNAs Function as Epigenetic Modulators in Nasopharyngeal Carcinoma. Front Oncol. 2019;9:1120.

25. Bose DA, Donahue G, Reinberg D, Shiekhattar R, Bonasio R, Berger SL. RNA Binding to CBP Stimulates Histone Acetylation and Transcription. Cell. 2017;1681-2:135-149.e122.

26. Cui W, Yoneda R, Ueda N, Kurokawa R. Arginine methylation of translocated in liposarcoma (TLS) inhibits its binding to long noncoding RNA, abrogating TLS-mediated repression of CBP/p300 activity. J Biol Chem. 2018;29328:10937-48.

27. Liu X, He F, Pang R, Zhao D, Qiu W, Shan KXet al. Interleukin-17 (IL-17)-induced microRNA 873 (miR873) contributes to the pathogenesis of experimental autoimmune encephalomyelitis by targeting A20 ubiquitin-editing enzyme. J Biol Chem. 2014;28942:28971-86.

28. Gao J, Kang XY, Sun S, Li L, Zhang BL, Li YQ『et al. Transcription factor Six2 mediates the protection of GDNF on 6-OHDA lesioned dopaminergic neurons by regulating Smurf1 expression. Cell Death Dis. 2016;75:e2217.

29. Zhou F, Liu X, Gao L, Zhou X, Cao Q, Niu L】et al. HIV-1 Tat enhances purinergic P2Y4 receptor signaling to mediate inflammatory cytokine production and neuronal damage via PI3K/Akt and ERK MAPK pathways. J Neuroinflammation. 2019;161:71.

30. Zhou F, Liu X, Zuo D, Xue M, Gao L, Yang Y『et al. HIV-1 Nef-induced IncRNA AK006025 regulates CXCL9/10/11 cluster gene expression in astrocytes through interaction with CBP/P300. J Neuroinflammation. 2018;151:303.

31. Liu X, Zhou F, Yang Y, Wang W, Niu L, Zuo D囚et al. MiR-409-3p and MiR-1896 co-operatively participate in IL-17-induced inflammatory cytokine production in astrocytes and pathogenesis of EAE mice via targeting SOCS3/STAT3 signaling. Glia. 2019;671:101-12.

32. Ponath G, Park C, Pitt D. The Role of Astrocytes in Multiple Sclerosis. Front Immunol. 2018;9:217.

33. Wang X, Mulas F, Yi W, Brunn A, Nishanth G, Just S囚et al. OTUB1 inhibits CNS autoimmunity by preventing IFN-y-induced hyperactivation of astrocytes. Embo j. 2019; 3810.

34. Bassil R, Orent W, Elyaman W. Notch signaling and T-helper cells in EAE/MS. Clin Dev Immunol. 2013;2013:570731. 
35. Fan H, Zhao JG, Yan JQ, Du GQ, Fu QZ, Shi J囚et al. Effect of Notch1 gene on remyelination in multiple sclerosis in mouse models of acute demyelination. J Cell Biochem. 2018;11911:9284-94.

36. Hosseini E, Bagheri-Hosseinabadi Z, De Toma I, Jafarisani M, Sadeghi I. The importance of long noncoding RNAs in neuropsychiatric disorders. Mol Aspects Med. 2019;70:127-40.

37. Cortini F, Roma F, Villa C. Emerging roles of long non-coding RNAs in the pathogenesis of Alzheimer's disease. Ageing Res Rev. 2019;50:19-26.

38. Sun D, Yu Z, Fang X, Liu M, Pu Y, Shao Q『et al. LncRNA GAS5 inhibits microglial M2 polarization and exacerbates demyelination. EMBO Rep. 2017;1810:1801-16.

39. Zhang F, Liu G, Wei C, Gao C, Hao J. Linc-MAF-4 regulates Th1/Th2 differentiation and is associated with the pathogenesis of multiple sclerosis by targeting MAF. Faseb j. 2017;312:519-25.

40. Yang X, Wu Y, Zhang B, Ni B. Noncoding RNAs in multiple sclerosis. Clin Epigenetics. 2018;101:149.

41. Ghoveud E, Teimuri S, Vatandoost J, Hosseini A, Ghaedi K, Etemadifar M『et al. Potential Biomarker and Therapeutic LncRNAs in Multiple Sclerosis Through Targeting Memory B Cells. Neuromolecular Med. 2020;221:111-20.

42. Ghaiad HR, Elmazny AN, Nooh MM, El-Sawalhi MM, Shaheen AA. Long noncoding RNAs APOA1-AS, IFNG-AS1, RMRP and their related biomolecules in Egyptian patients with relapsing-remitting multiple sclerosis: Relation to disease activity and patient disability. J Adv Res. 2020;21:141-50.

\section{Figures}


A
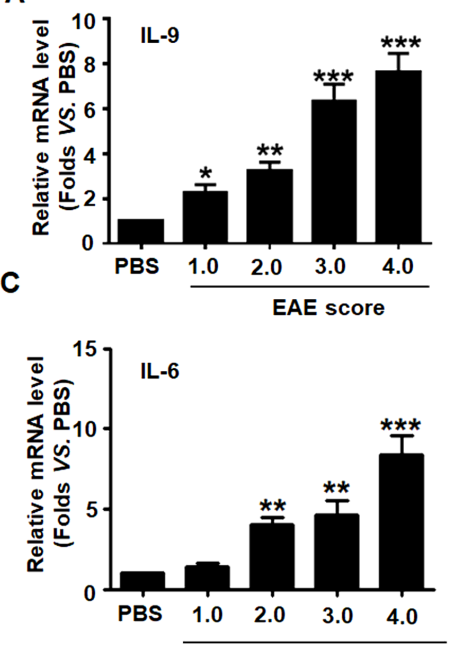

D

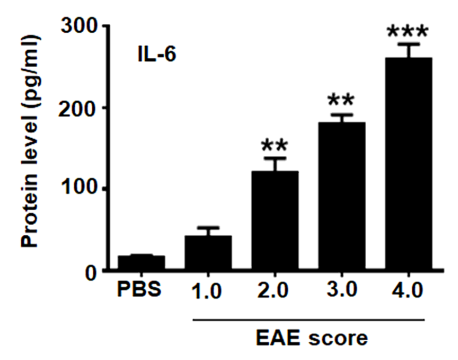

B
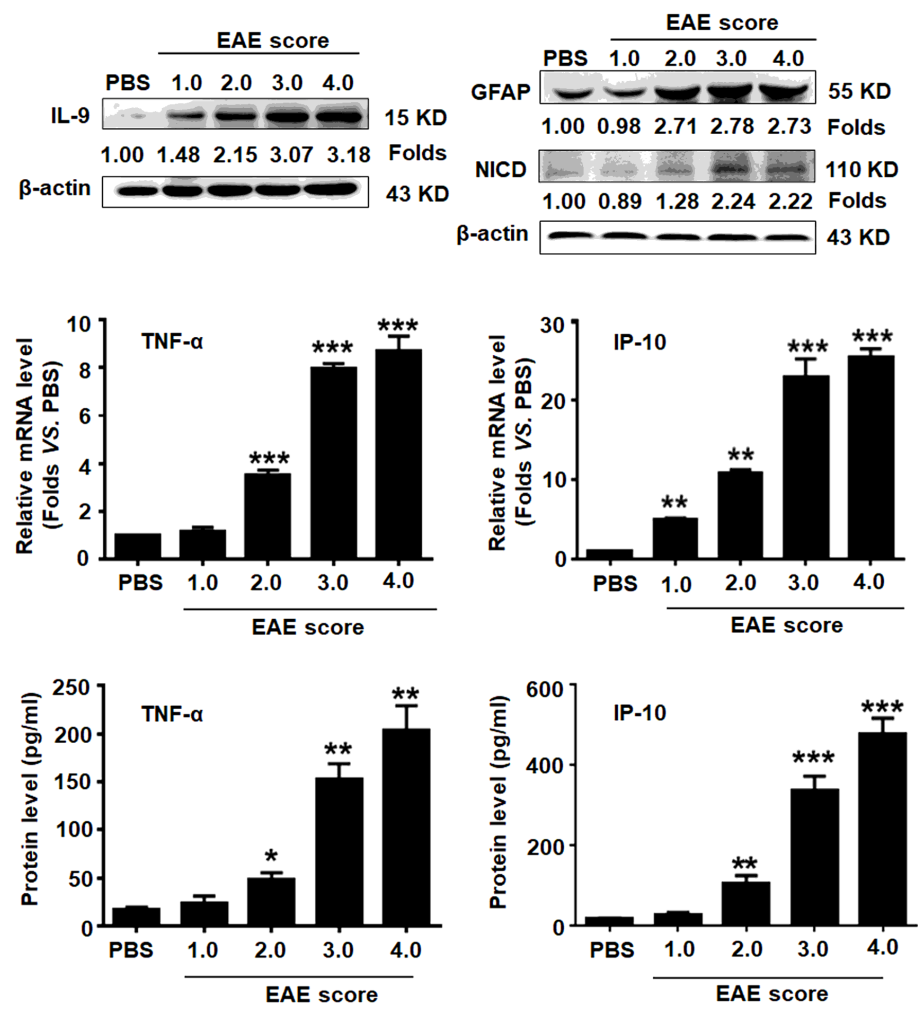
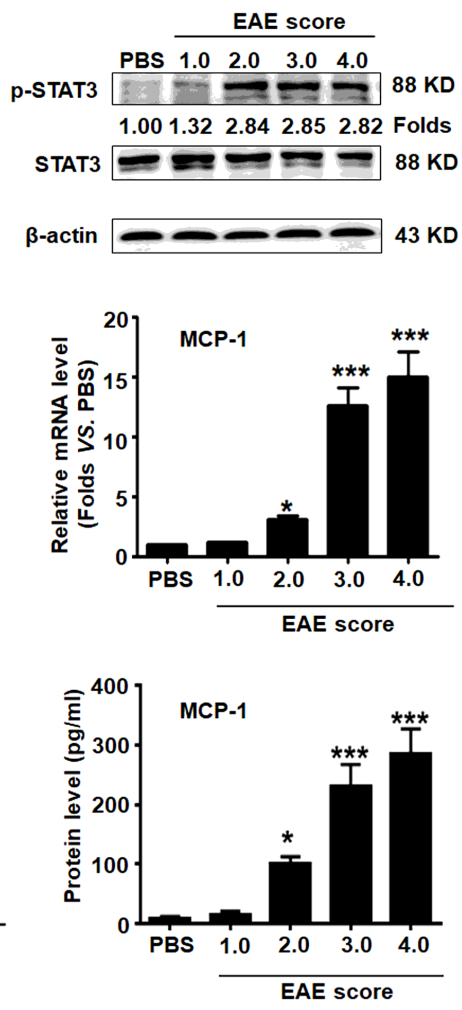

Figure 1

Upregulation of IL-9 and inflammatory cytokines as well as the activation of Notch1 signaling during EAE process. a The level of IL-9 in spinal cords of EAE mice with different clinical score was measured by realtime PCR and Western blot assay, respectively. b The expressions of GFAP, NICD and p-STAT3 in spinal cords along with EAE process were detected by Western blot assay. c The mRNA levels of IL-6, TNF-a, IP10 and MCP-1 in spinal cords were determined using real-time PCR. $d$ The secretion levels of IL-6, TNF-a, IP-10 and MCP-1 in the sera were detected by Cytometric Bead Array (CBA) during EAE process. *p $<0.05$, ${ }^{* *} \mathrm{p}<0.01$ and ${ }^{* *} \mathrm{p}<0.001$ versus PBS group ( $\mathrm{n}=6 /$ group). Data are represented as the mean \pm SEM. 
A
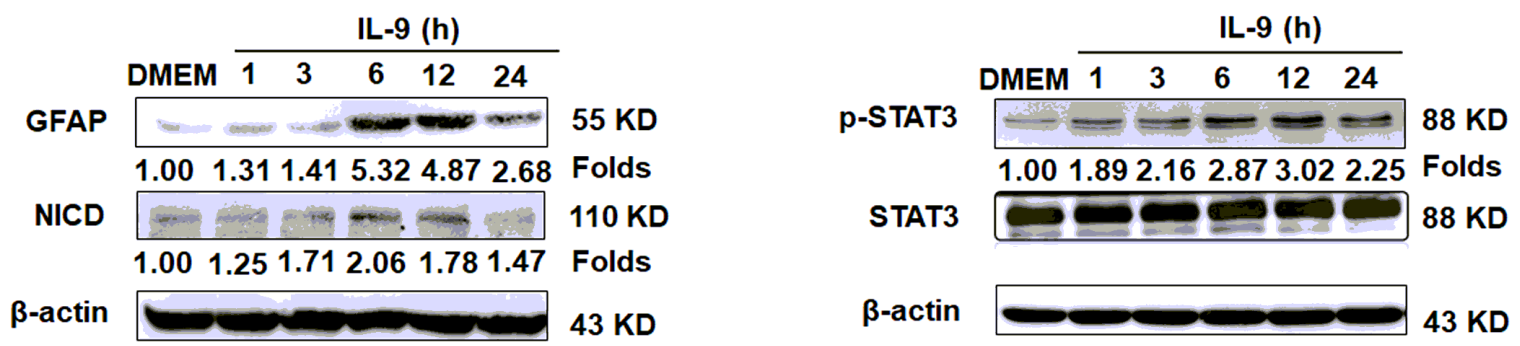

B

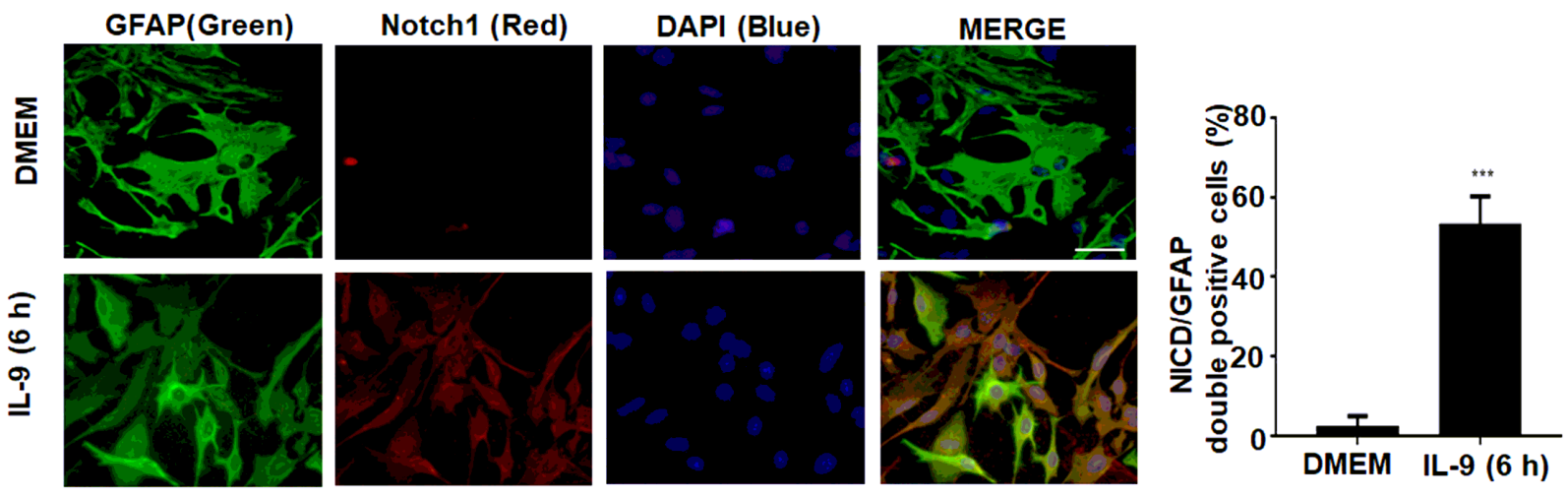

C
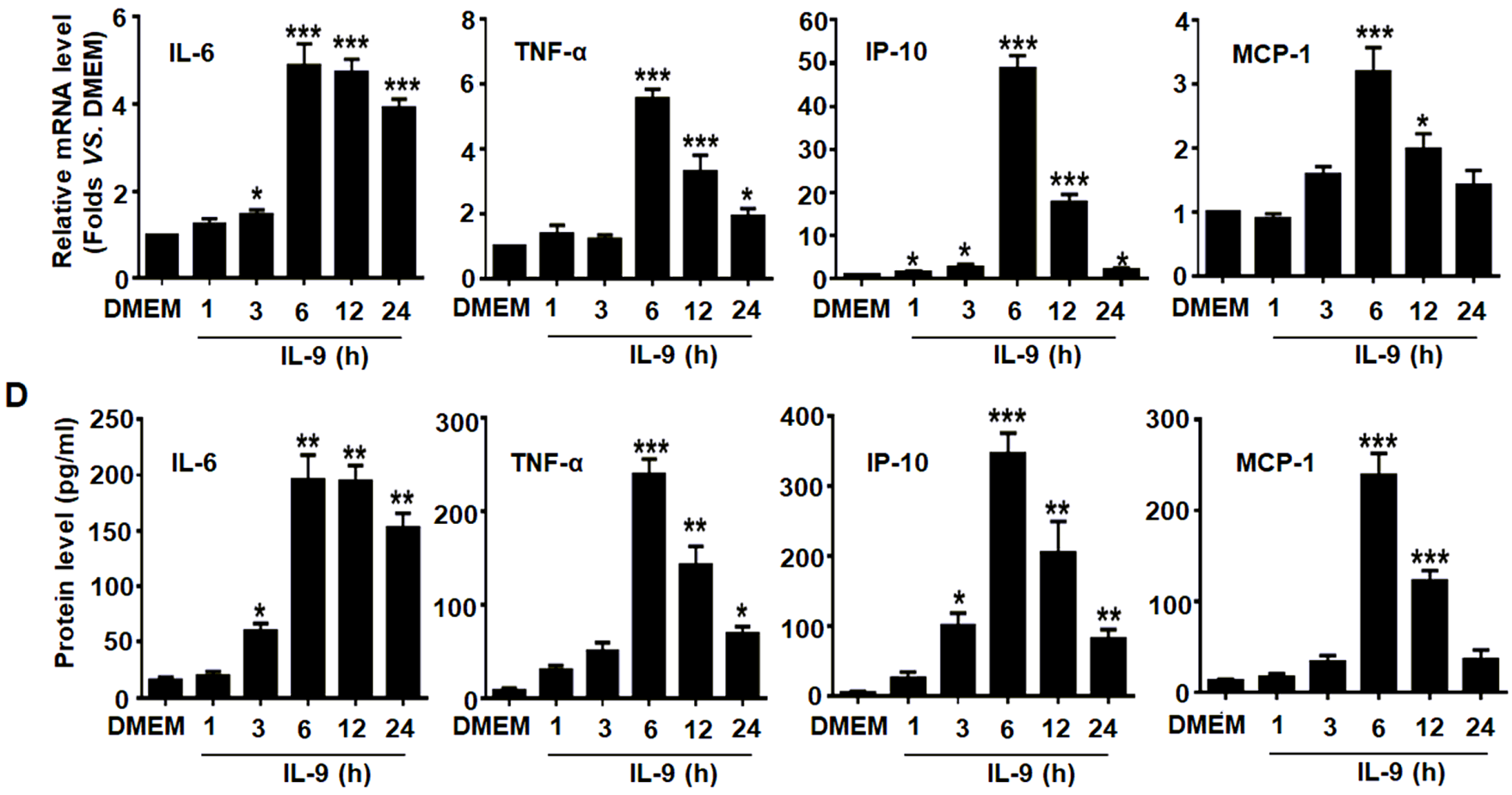

Figure 2

IL-9 activates Notch1 pathway and promotes inflammatory cytokines production in astrocytes. Primary mouse astrocytes were incubated in a serum-free medium overnight followed by treating with IL-9 at indicated time point. a The expression changes of GFAP, NICD and p-STAT3 were analyzed by Western blot assay. b Immunofluorescent staining for GFAP (green), Notch1 (red) and nuclear staining of DAPI (blue) in cultured astrocytes with IL-9 treatment for $6 \mathrm{~h}$. Scale bars, $50 \mu \mathrm{m}$. c The mRNA levels of IL-6, 
TNF-a, IP-10 and MCP-1 in astrocytes were detected using real-time PCR assay. $d$ The secretion levels of IL-6, TNF-a, IP-10 and MCP-1 in the supernatant of astrocytes were measured by CBA assay. ${ }^{*} p<0.05$, ** $p$ $<0.01$ and ${ }^{* \star *} \mathrm{p}<0.001$ versus DMEM group. The Data are from three independent experiments and represented as the mean \pm SEM.

A

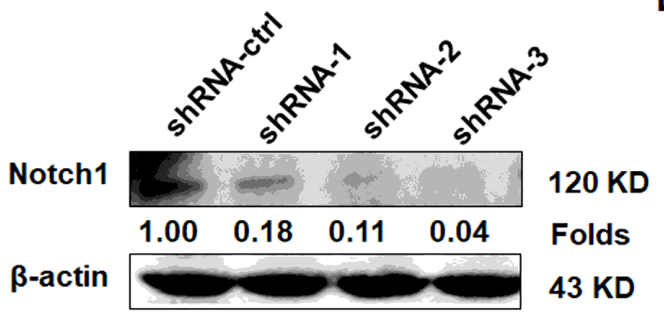

B

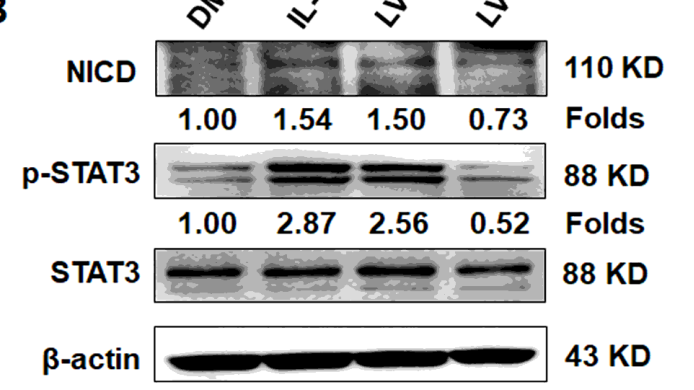

C

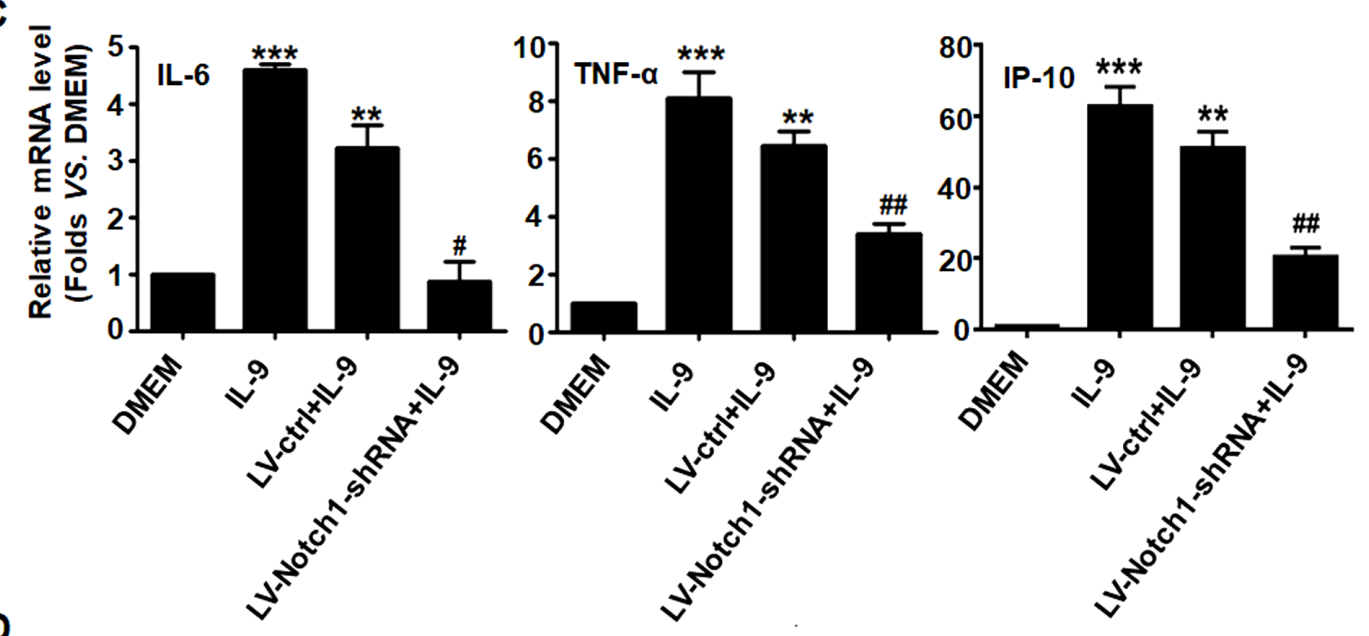

D
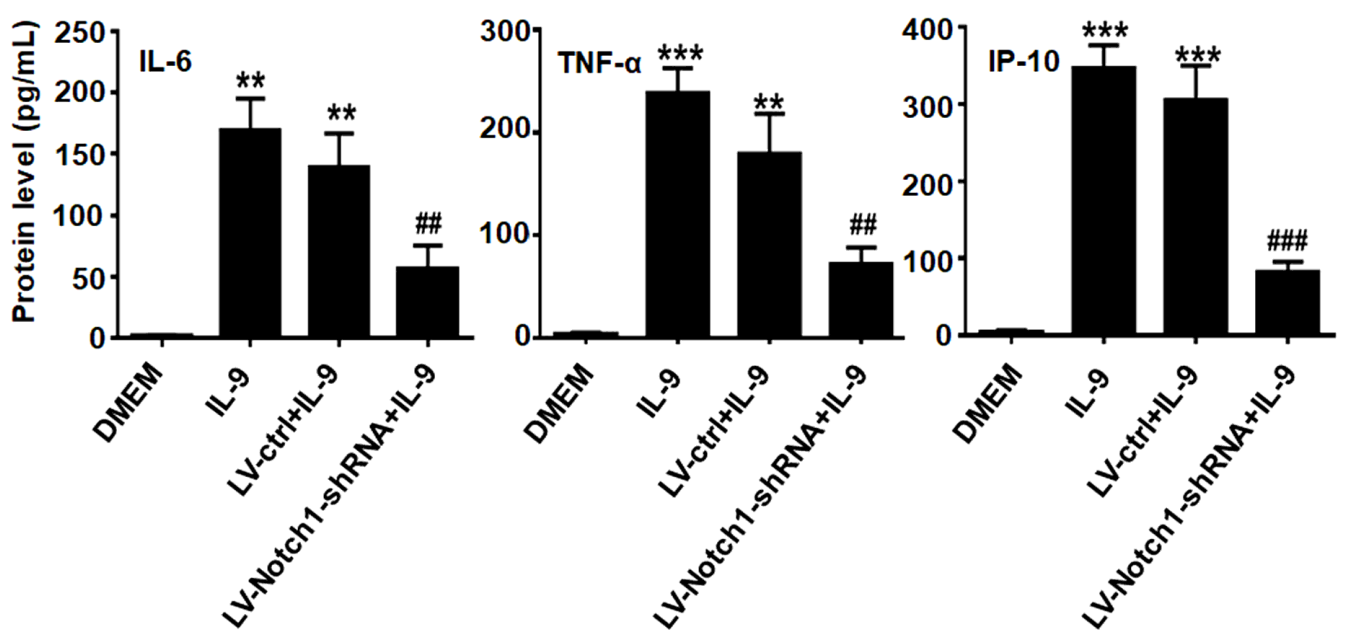

Figure 3 
Knockdown of Notch1 reduces inflammatory cytokines production in activated-astrocytes by IL-9. a The 3 recombinant lentivirus plasmids of Notch1-shRNA targeting astrocytes were transduced into mouse primary astrocytes using the Neon ${ }^{\mathrm{TM}}$ electron transfection system (MPK5000) for $48 \mathrm{~h}$. The expression of Notch1 protein in astrocytes was evaluated by Western blot assay. b Primary mouse astrocytes were infected with recombinant lentivirus (LV-Notch1-shRNA, LV-ctrl) for $72 \mathrm{~h}$, and then incubated in a serumfree medium overnight followed by treating with IL-9 for $6 \mathrm{~h}$. Western blot analysis for the proteins of Notch1 pathway. c The mRNA levels of IL-6, TNF- $a$ and IP-10 in astrocytes were measured by real-time PCR. $d$ The secretion levels of IL-6, TNF- $a$ and IP-10 in the supernatant of astrocytes were detected by CBA assay. ${ }^{* \star} p<0.01$ and ${ }^{* \star *} p<0.001$ versus DMEM group; $\# p<0.05$ and $\# \# p<0.01$ and \#\#\#p $<0.001$ versus LV-ctrl+IL-9 group. The Data are from three independent experiments and represented as mean \pm SEM.

A

The sequence of IncRNA Gm13568 $\mathrm{t} t \mathrm{t} t \mathrm{t} t \mathrm{tg}$ TTTAGCAAAATGTGGACAAOCATCAGTATCATTTTTATTGCAAATTAGTTAHCAAAAAAA GATGAHAHAHATACATCATTTTCATTACCTACAGTTTTGCATGTAGTTTTTTTTTTTACGTTTCTTTT TTTTGTAAACTACACTTTATTTTATACAACACAGTAAGAATCAACATCTTAGGATGCGTCTGGTCATAT CTATTAGCCATGGCACAGACACTGCOCCOCAAAAGGCCAGAAAGAGCTGOCCTGAGGAGGTGGGCAACT GOCCOCGAAACCCOCCAGCACACAGTGGCTGGAAAGGGACTTGGTGGGCAGCAGATGGGCCATCCTGGG TTGTCCTCTTAGGAGCTACTGGTTCGGCAGCTACAGGTCACAAGGTCTGGGTCACACTGAGCTAAGCCA CAGTCTGAC

C

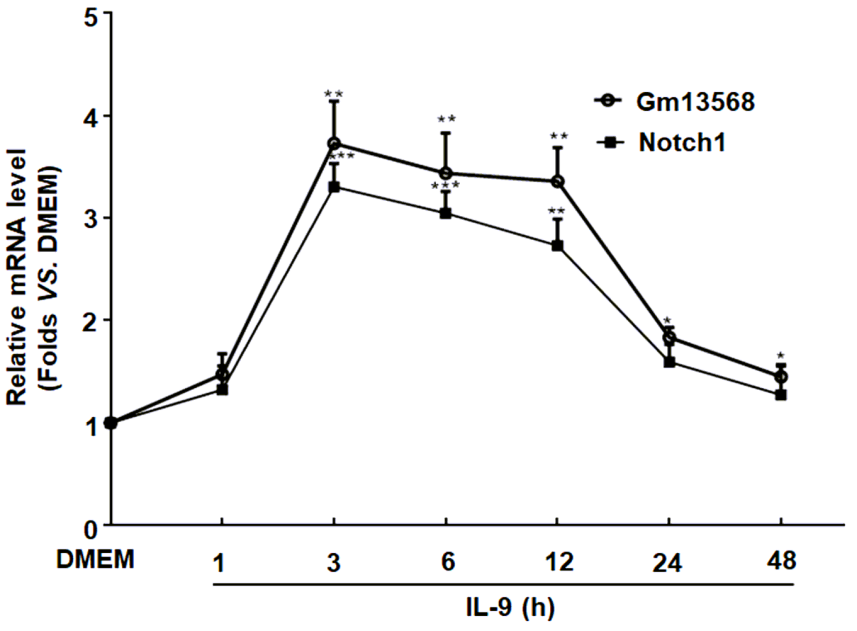

B

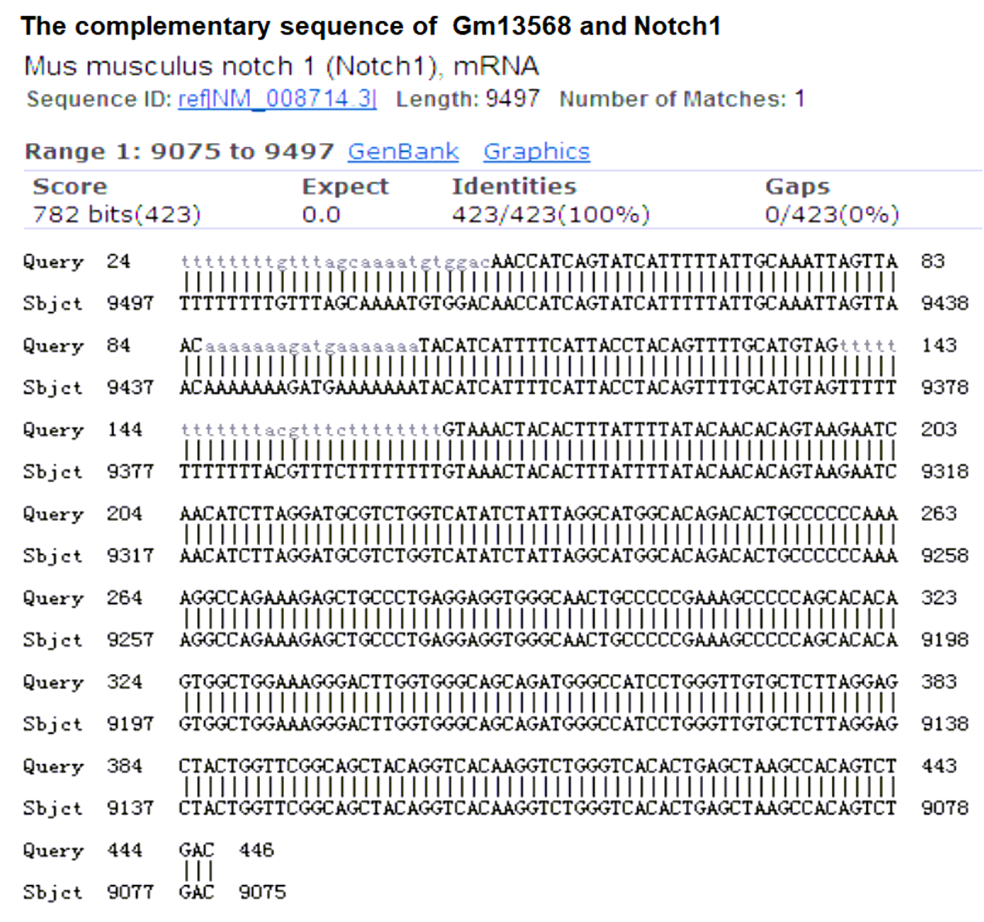

\section{Figure 4}

Identification of IncRNA targeting Notch1 gene. a The sequence of IncRNA Gm13568 with 446 base length. b The sequence of Gm13568 is complementary to the sense chain of Notch1 gene at 9075-9497 locus. c Primary mouse astrocytes were treated in a serum-free medium overnight followed by stimulating with IL-9 at different time period. Real-time PCR was performed to detect the change levels of Gm13568 and Notch1 in astrocytes. ${ }^{*} p<0.05$ and ${ }^{*} p p<0.01$ and ${ }^{* \star *} p<0.001$ versus DMEM. Results are represented as mean \pm SEM. 
A

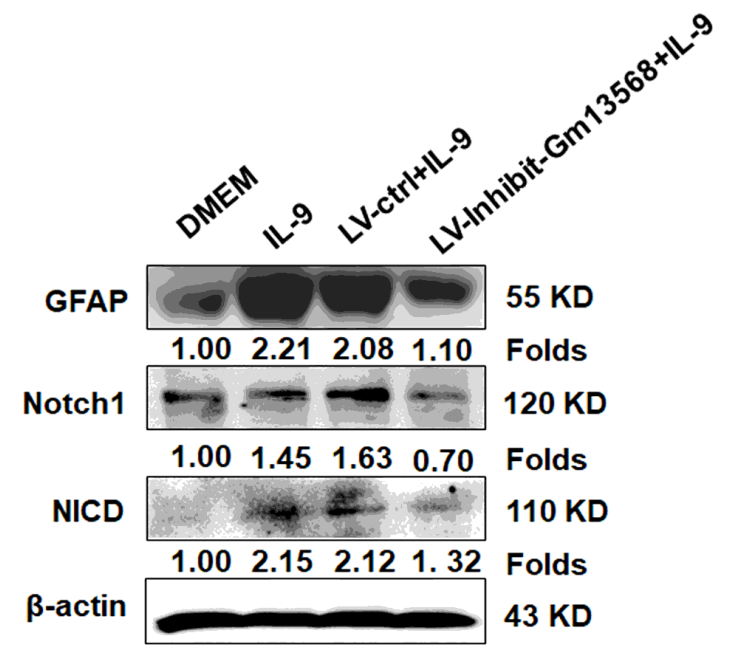

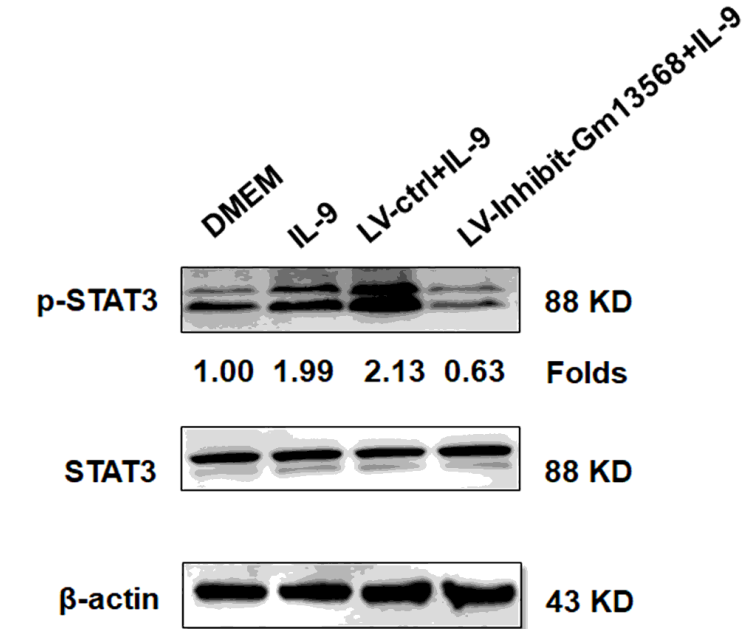

B
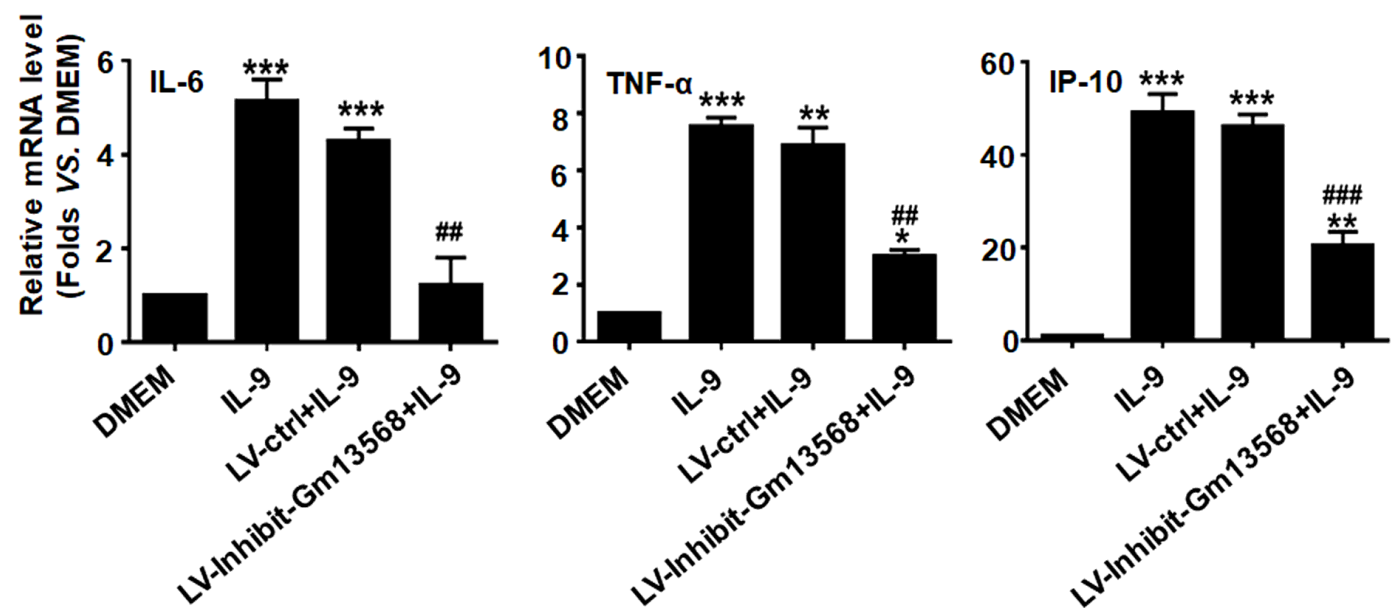

C
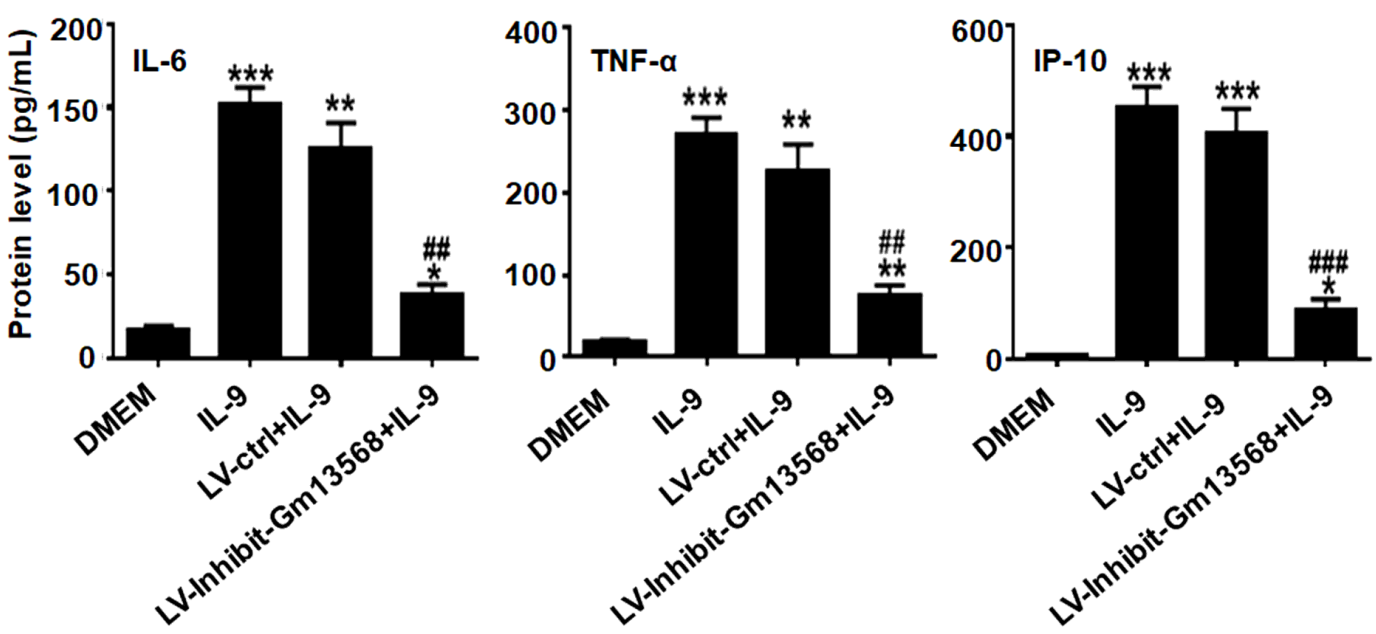

Figure 5

Inhibition of Gm13568 downregulates Notch1 signaling activation as well as inflammatory cytokines production in astrocytes by IL-9. a Primary mouse astrocytes were infected with recombinant lentivirus, LV-Inhibit-Gm13568 and LV-ctrl, for $72 \mathrm{~h}$, respectively. Then, the astrocytes were incubated in a serum-free medium overnight followed by IL-9 stimulation for $6 \mathrm{~h}$. Western blot assay was used for measuring the protein expressions of GFAP, Notch1/NICD and p-STAT3. b The mRNA levels of IL-6, TNF- $a$ and IP-10 in 
astrocytes were measured by real-time PCR assay. c The secretion of IL-6, TNF- $a$ and IP-10 in the supernatant of astrocytes were analyzed by CBA assay. The Data are represented as the mean \pm SEM. * $p$ $<0.05,{ }^{* *} p<0.01$ and ${ }^{* \star *} p<0.001$ versus DMEM group; \#\#p $<0.01$ and \#\#\#p $<0.001$ versus LV-ctrl+IL-9 group.

A
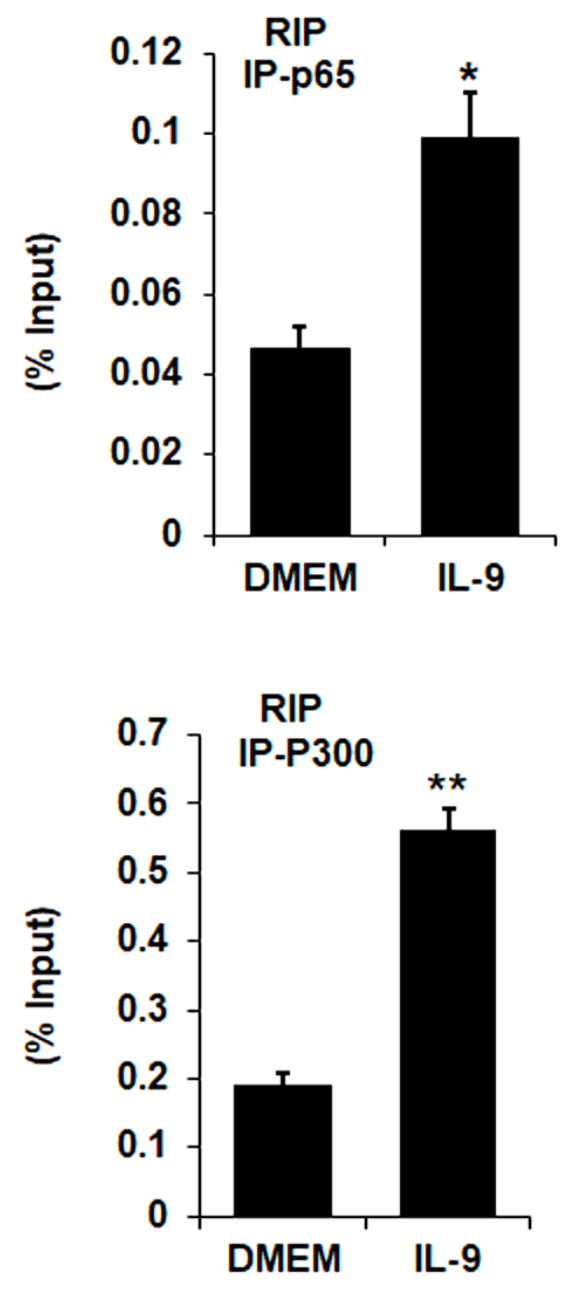

B

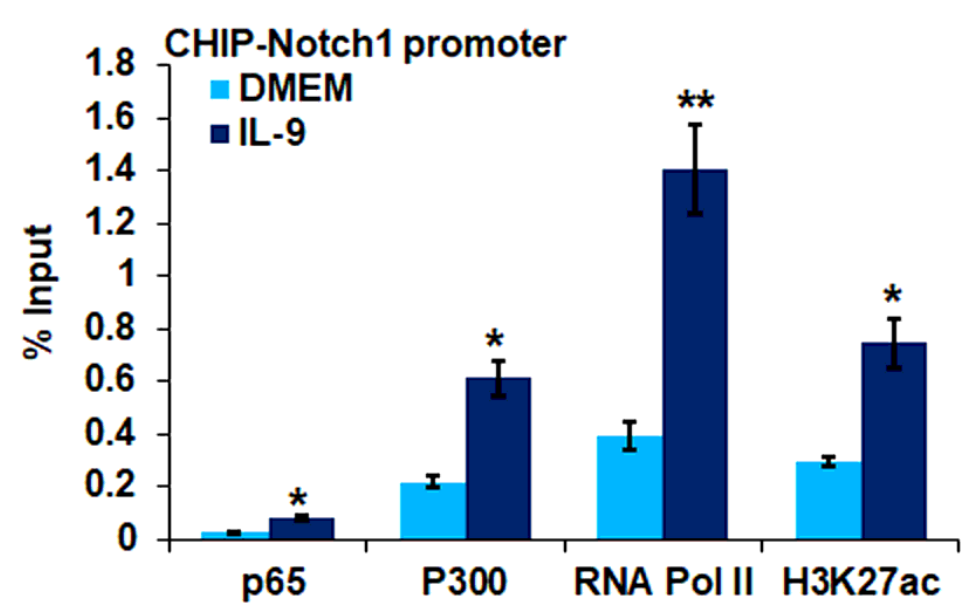

C

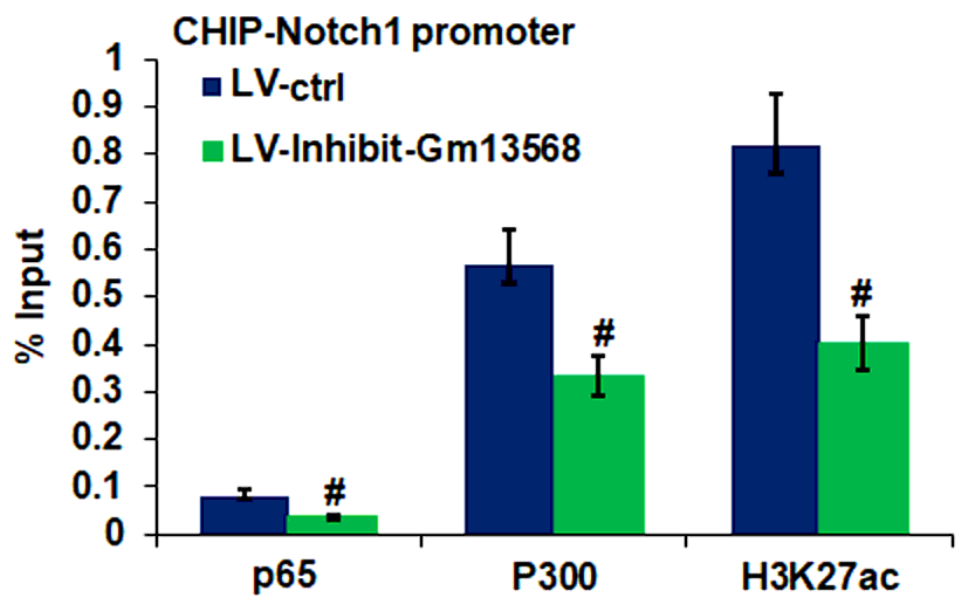

Figure 6

LncRNA Gm13568 interacts with CBP/P300 to regulate the expression of Notch1 in astrocytes. a RIP assay was performed for the interaction of Gm13568 with NF-KB p65 and CBP/P300 in astrocytes treatment with IL-9 for $6 \mathrm{~h}$. The data were from three independent experiments. $\mathrm{b}$ ChIP assay analysis of NF-KB p65, CBP/P300, RNA Pol II, and H3K27ac enrichment on the promoter of Notch1 gene in astrocytes stimulation with IL-9 for 6 h. ${ }^{*} p<0.05, * \star p<<0.01, * \star \star p ~<0.001$ vs DMEM. Error bars represent mean \pm SEM. c ChIP assay analysis of NF-KB p65, CBP/P300 and H3K27ac enrichment on the promoter of Notch1 gene in astrocytes infected by LV-Inhibit-Gm13568 for 72 h, followed by stimulation with IL-9 for 6 h. $\# p<0.05$ versus LV-ctrl. The data were from three independent experiments. 
A

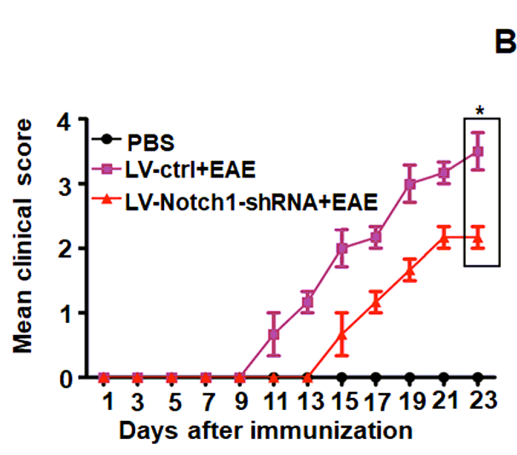

B

C

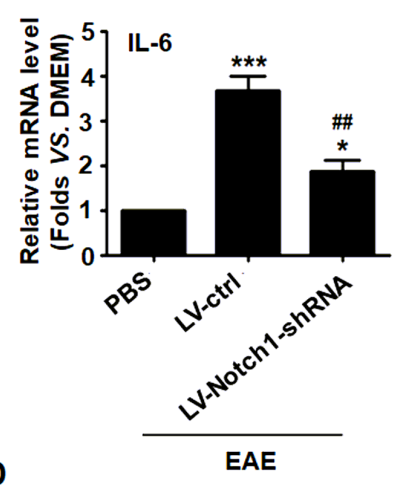

D

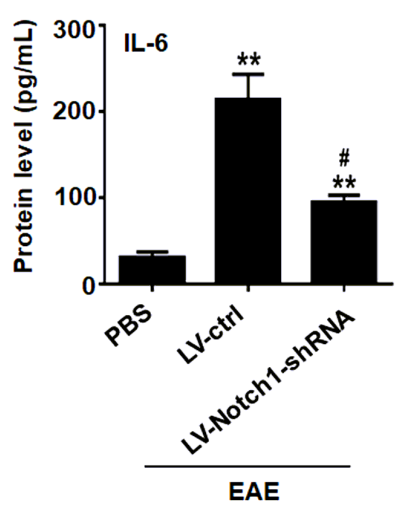

E

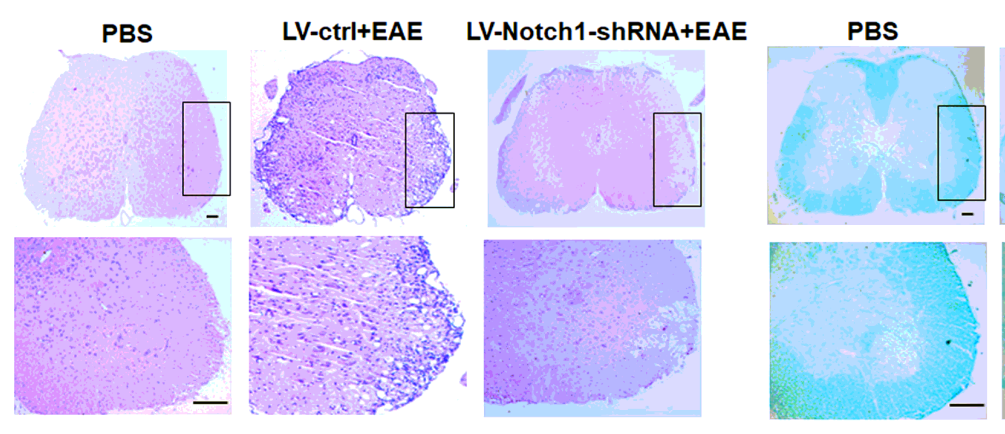

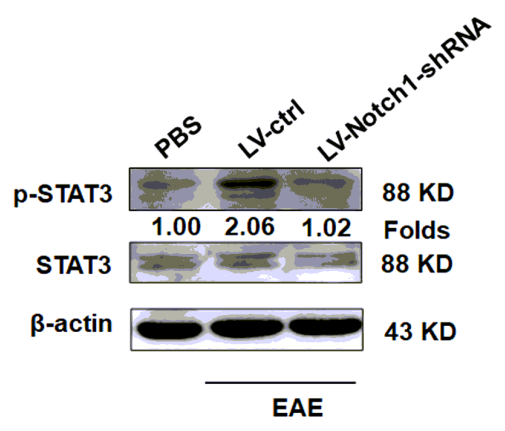
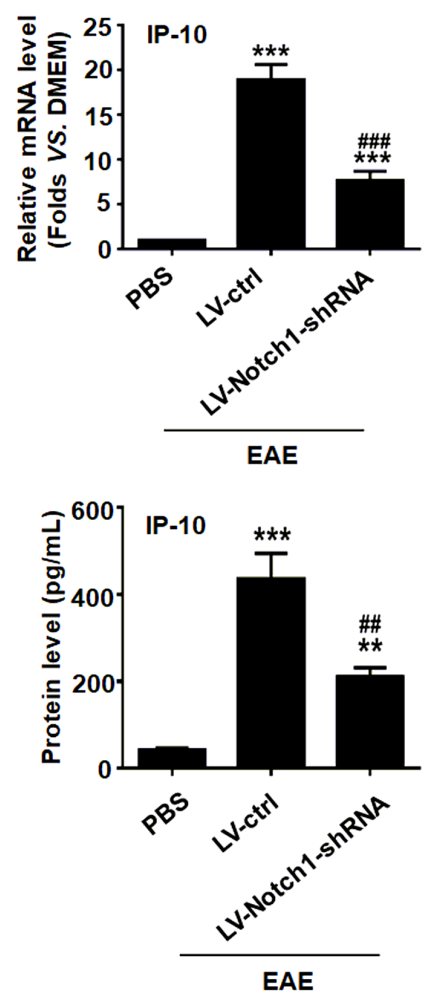

LV-ctrl+EAE LV-Notch1-shRNA+EAE

\section{Figure 7}

Knockdown of Notch1 in astrocytes suppresses inflammation and alleviates EAE in mice. Mice were subjected to recombinant lentiviruses, LV-ctrl or LV-Notch1-shRNA, for $7 \mathrm{~d}$, followed by M0G35-55 immunization for $23 \mathrm{~d}$ ( $\mathrm{n}=10$ mice per group). a The clinical scores of EAE mice with LV-ctrl and LVNotch1-shRNA. b The expressions of IL-9, GFAP, NICD and p-STAT3 in the spinal cords were detected using western blot assay. c, $d$ The changes of IL-6, TNF- $a$ and IP-10 in the spinal cords and peripheral 
blood of the LV-ctrl and LV-Notch1-shRNA mice were evaluated by real-time PCR and CBA assay,

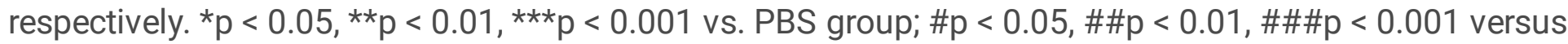
LV-ctrl group. Results are represented as mean \pm SEM. e The infiltrations of inflammatory cells in spinal cords were investigated using Hematoxylin and Eosin (H\&E) staining (Scale bars, $50 \mu \mathrm{m}$ ). $f$ The medullary

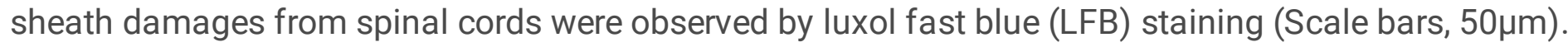
Boxed areas in the upper rows are presented enlarged underneath.

A

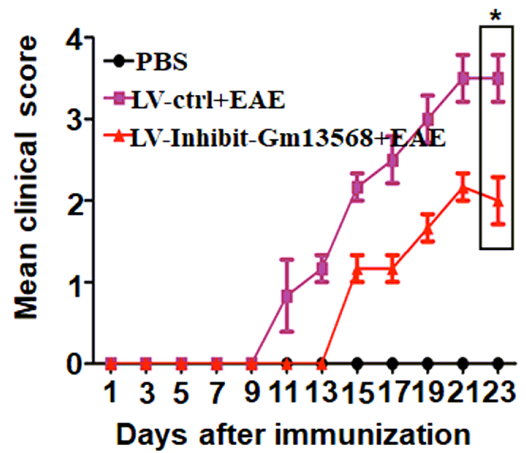

B
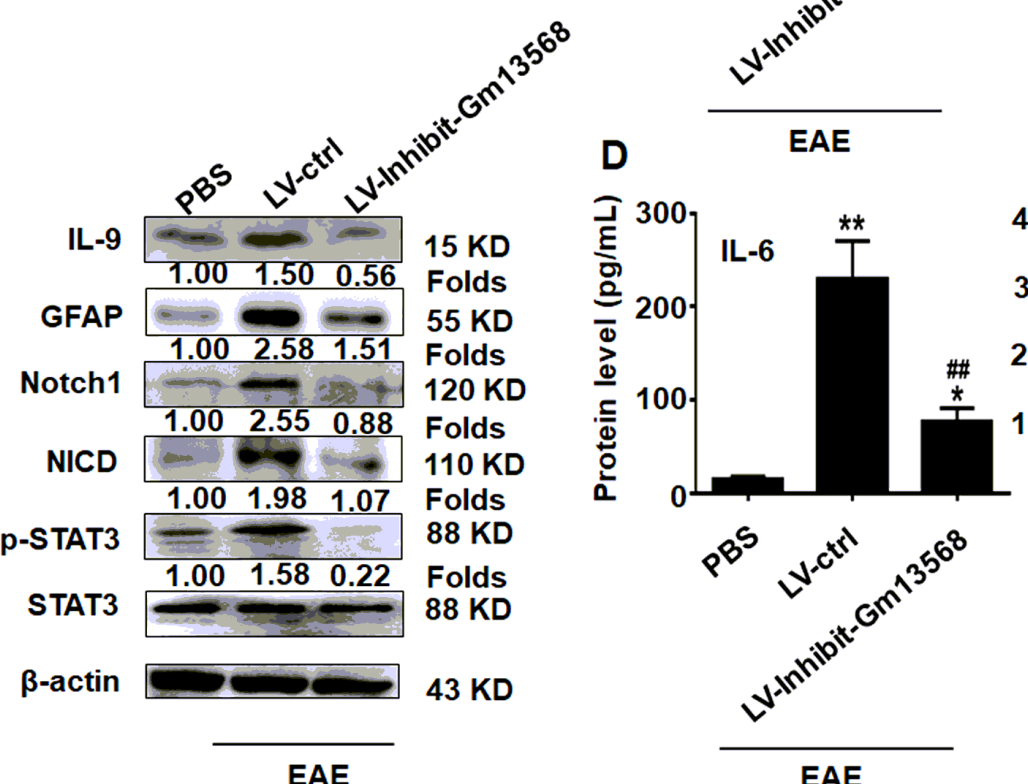

$\mathbf{E}$ PBS
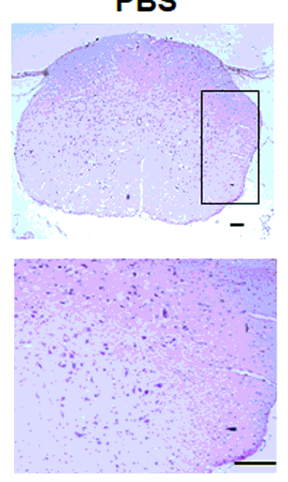

C
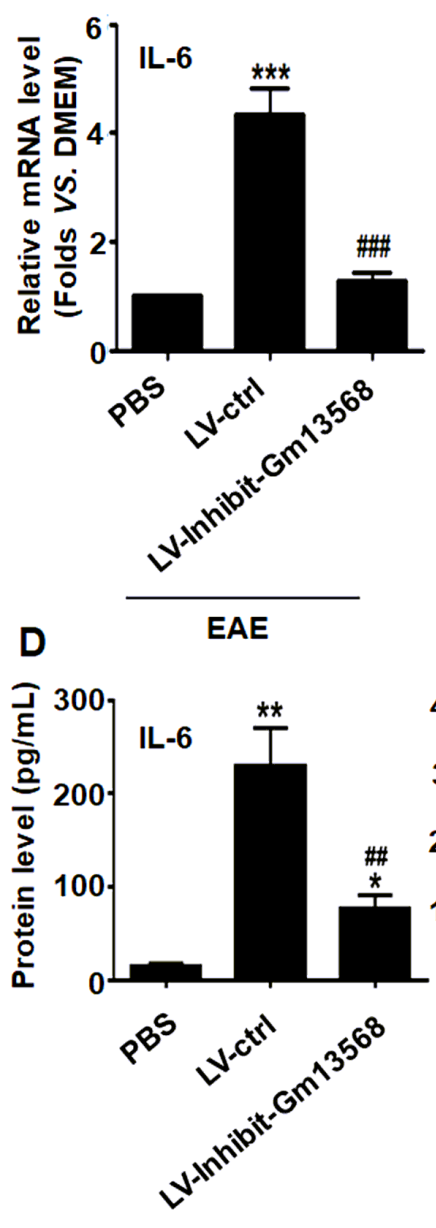

EAE

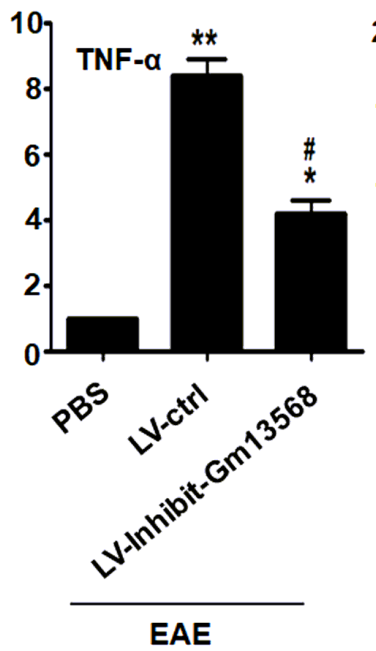

EAE

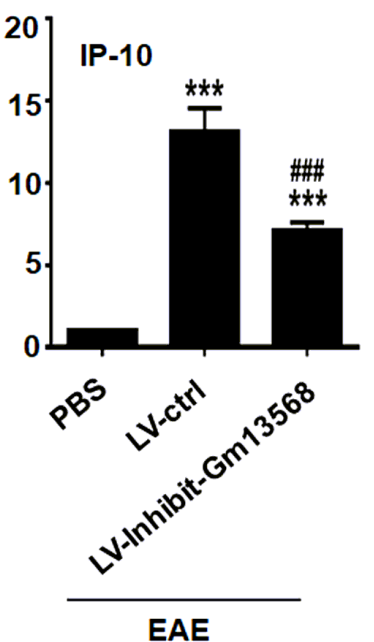

EAE

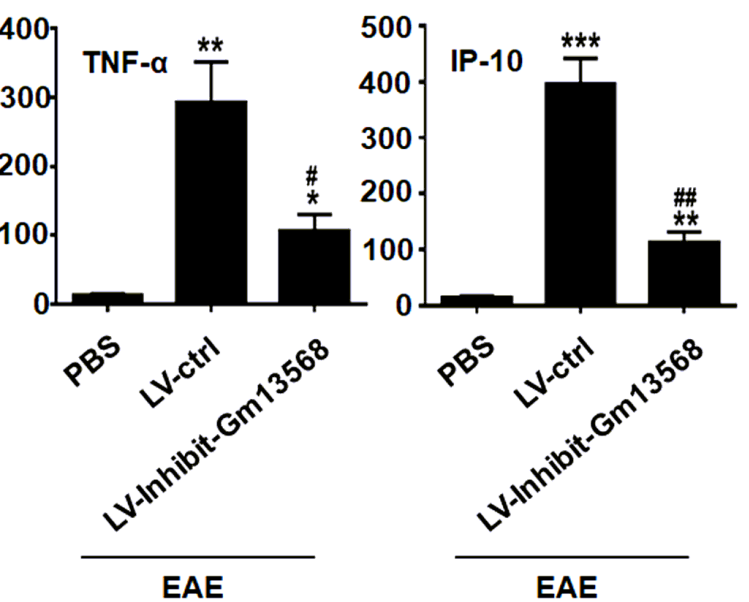

PBS

LV-ctrl+EAE

LV-Inhibit-Gm13568+EAE

Figure 8 
The inhibition of Gm13568 in astrocytes decreases Notch1 pathway activation and ameliorates pathological process in EAE mice. On day 7 after administration with LV-ctrl or LV-Inhibit Gm13568, the mice were immunized with MOG35-55 for $23 \mathrm{~d}$. a The clinical scores for EAE mice infected by LV-ctrl or LV-Inhibit Gm13568 ( $\mathrm{n}=10$ mice per group). $b$ The expressions of IL-9 and GFAP and the activation of Notch1 pathway in the spinal cords were evaluated by Western blot assay. The results of a typical experiment are presented. c, $d$ The levels of IL-6, TNF- $a$ and IP-10 from the spinal cords and peripheral blood in lentivirus-infected mice were analyzed by real-time PCR and CBA assay, respectively. ${ }^{*} p<0.05$,

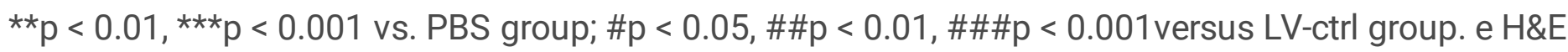
staining for inflammatory cells infiltrations in spinal cords of mice (Scale bars, $50 \mu \mathrm{m}$ ). $\mathrm{f}$ LFB staining for the medullary sheath damage in spinal cords of mice (Scale bars, $50 \mu \mathrm{m}$ ). Boxed areas in the upper rows are presented enlarged underneath.

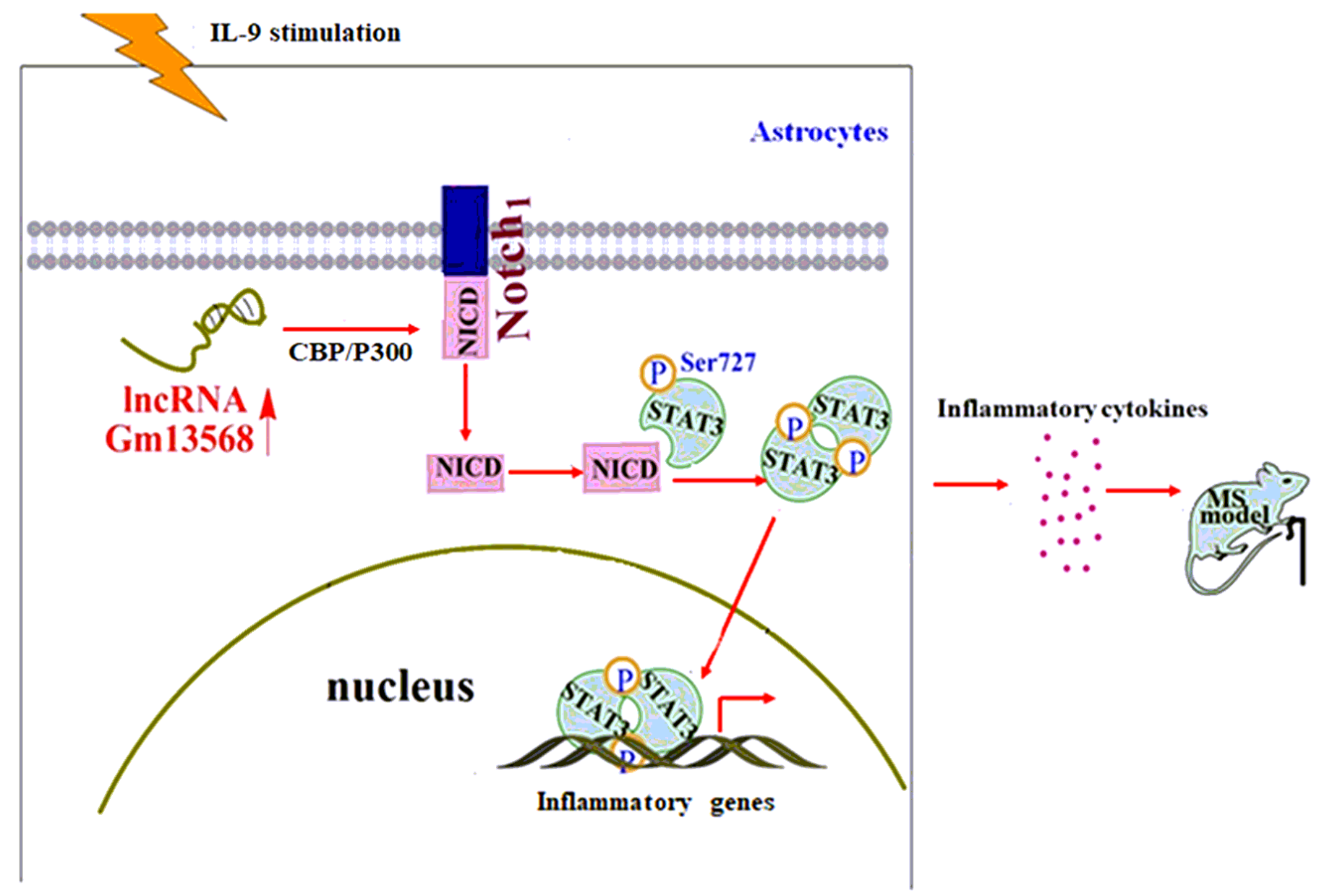

Figure 9

Schematic representation of IncRNA Gm13568 participating the pathogenesis of EAE mice through regulating Notch1 pathway. Under IL-9 stimulation, Gm13568 is upregulated in astrocytes, which promotes to the expression and activation of Notch1 via interacting with CBP/P300, in turn contributes to the release of inflammatory cytokines, thus aggravating the EAE process. 


\section{Supplementary Files}

This is a list of supplementary files associated with this preprint. Click to download.

- SupplFig.1.tif

- SupplTable1.PrimersequencesforqPCR.doc 